\title{
Oscillations and variability in neuronal systems: interplay of autonomous transient dynamics and fast deterministic fluctuations
}

\author{
Rodrigo F. O. Pena, Horacio G. Rotstein *广 \\ Federated Department of Biological Sciences \\ New Jersey Institute of Technology and Rutgers University, USA
}

June 14, 2021

\begin{abstract}
Neuronal systems are subject to rapidly fluctuations both intrinsically and externally. In mathematical models, these fluctuations are typically incorporated as stochastic noise (e.g., Gaussian white or colored noise). Noise can be both disruptive and constructive, for example, by creating irregularities and variability in otherwise regular patterns or by creating oscillatory patterns and increasing the signal coherence, respectively. The dynamic mechanisms underlying the interactions between rapidly fluctuating signals and the intrinsic properties of the target cells to produce variable and/or coherent responses are not fully understood. In particular, it is not clear what properties of the target cell's intrinsic dynamics control these interactions and whether the generation of this phenomena requires stochasticity of the input signal and, if yes, to what degree. In this paper we investigate these issues by using linearized and non-linear conductance-based models and piecewise constant (PWC) inputs with short duration pieces and variable amplitudes, which are arbitrarily, but not necessarily stochastically distributed. The amplitude distributions of the constant pieces consist of arbitrary permutations of a baseline PWC function with monotonically increasing amplitudes. In each trial within a given protocol we use one of these permutations and each protocol consists of a subset of all possible permutations, which is the only source of uncertainty in the protocol. We show that sustained oscillatory behavior can be generated in response to additive and multiplicative PWC inputs in both linear and nonlinear systems, independently of whether the stable equilibria of the corresponding unperturbed systems are foci (exhibiting damped oscillations) or nodes (exhibiting overshoots). The oscillatory responses are amplified by the model nonlinearities and attenuated for conductance-based PWC inputs as compared to current-based PWC inputs, consistent with previous theoretical and experimental work. In addition, the responses to PWC inputs exhibited variability across trials, which is reminiscent of the variability generated by stochastic noise (e.g., Gaussian white noise). This variability was modulated by the model parameters and the type of cellular intrinsic dynamics. Our analysis demonstrates that both oscillations and variability are the result of the interaction between the PWC input and the autonomous transient dynamics with little to no contribution from the dynamics around the steady-state. The generation of oscillations and variability does not require input stochasticity, but rather the sequential activation of the transient responses to
\end{abstract}

*E-mail: horacio@njit.edu, corresponding author

${ }^{\dagger}$ Corresponding Investigator, CONICET, Argentina. 
abrupt changes in constant inputs. Each piece with the same amplitude evokes different responses across trials due to the differences in initial conditions in the corresponding regime. These initial conditions are determined by the value of the voltage at the end of the previous regime, which is different for different trials. The predictions made in this papers are amenable for experimental testing both in vitro and in vivo.

\section{Introduction}

The response of dynamical systems having relatively simple attractors to external inputs consist of a transient phase followed by the approximation to the stationary solution (e.g., equilibrium, limit cycle). Both phases contain information about the model parameters and, to a higher or lesser degree, both encode information about the input (see Appendix A.3), but knowledge of one does not necessarily provide unambiguous information about the other. For one-dimensional linear systems receiving a constant input, for example, one needs to know the input value in order to be able to predict the system's time constant (reciprocal of the eigenvalue) from the steady-state value. For two-dimensional linear systems, the parameter degeneracy is stronger and even if the number of parameters is reduced, computing the eigenvalues from knowledge about the steady state values is not possible (see Appendix A.3).

The investigation of dynamical systems primarily focuses on stationary solutions [1-3] and the properties of the transient behavior are less explored or even overlooked. Attractor networks are a key concept in neural computation [4-10] and have been proposed to capture a variety of cognitive process (e.g., working memory, navigation). Recently, transient dynamics has been argued to be important for neuronal processing and the encoding of information [11-17].

Time-dependent inputs can be approximated by a discrete sequence of short-duration constant pieces (e.g., Fig. 1-a) and therefore the sequence of the properly joined transient solutions to these constant pieces (Figs. 1-c to -e) provides an approximation to the system's response to the original (time-dependent) inputs. The short duration of the constant pieces does not allow for the corresponding steady-state solutions to develop and therefore the information about the input is encoded by the transient trajectories. Complex odors (different types of stimuli at different concentrations) and their representations have been proposed to operate in a similar way $[11,17]$.

The transient dynamics of individual cells (autonomous transient dynamics), which are uncovered by their response to abrupt changes in constant inputs, are key to understand the response of these cells to oscillatory inputs [18-20]. A prototypical example is given by subthreshold resonance. Subthreshold resonance refers to the ability of cells to exhibit a peak in the impedance amplitude profile (curve of the impedance amplitude as a function of the input frequency, Figs. 2-b3 and c3) for a preferred (resonant) input frequency [21,22] (see Appendix A). Subthreshold resonance emerges in both cells having intrinsic oscillatory properties (e.g., two-dimensional linear cells with complex eigenvalues, Fig. 2-c3) and cells lacking intrinsic oscillatory dynamics [18, 23] (e.g., twodimensional linear cells having real eigenvalues, , Fig. 2-b3), but exhibiting an overshoot (or sag). Because resonance and intrinsic oscillations can occur in the absence of each other and when they coexist the intrinsic (natural) and resonant frequencies do not generally coincide, the information provided by the eigenvalues is not enough to understand the mechanisms of resonance generation and amplification. The effective time scale responsible for the overshoot or the initial portion of the oscillation in response to constant inputs (autonomous transient dynamics) is key for the generation of resonance in response to oscillatory inputs $[18,19]$. The resonant frequency is determined as the optimal balance between the speed of the cyclically "moving voltage nullcline" in the extended phase-plane diagram and the effective time scale governing the evolution of the response trajectory tracking the motion of the voltage nullcline, which can be thought of as a continuous sequence of transient responses to the continuously changing input target $[19,20]$.

Neurons and neuronal networks are subject to intrinsic random fluctuations [24-26] such as 
random opening/closing of ionic channels [27-34] and random synaptic inputs from other neurons in the network [35-41]. Random fluctuations are also exerted by external factors. In mathematical models, these fluctuations are incorporated as noise interacting in various ways with the underlying deterministic dynamics [42].

Noise plays many roles in the resulting patterns. It can uncover the transient dynamics of dynamical systems [43]. It can also modify the dynamics prescribed by the underlying deterministic (mean field) system [44], for example, by creating sustained (irregular) oscillations in systems that would exhibit an equilibrium otherwise [45] or by creating bursting (spiking) patterns in systems that would exhibit deterministic spiking (bursting) patterns otherwise.

While from one point of view, noise is disruptive in the sense that it creates irregularities in otherwise regular patterns or even destroys equilibrium patterns, from another perspective, noise is constructive in the sense that it may create oscillatory patterns or increase the signal coherence. Two prototypical examples are stochastic resonance (a maximal amplitude response of a nonlinear system when is simultaneously driven by a weak periodic signal and noise for certain noise amplitude) [46-54] and coherence resonance (a maximum characteristic correlation time of a noise-driven oscillation for a certain noise amplitude, at some noise amplitude the regularity of the process is maximal in the absence of any periodic forcing) [55-62] (and references therein). The related phenomenon of stochastic resonance without periodic forcing [63-65] uncovers the effect of noise when the systems is in a regime near a bifurcation point. Additionally, noise can induce order in chaotic dynamics [66], promote synchronization (stochastic synchronization) [67-69] or generate synchronized oscillations in networks of coupled excitable elements [70-73], promote the generation of slow wave oscillations by inducing transitions between active and silente phases [74,75], and induce transitions in bistable and excitable systems (non-oscillatory) [31,61,76-80] among other phenomena.

Common to these phenomena is the idea that noise repeatedly "kicks" trajectories outside their stationary solutions to create alternative patterns (irregular versions of the underlying noiseless pattern or qualitatively different patterns). In practice, noise interacts with the cell, which responds by integrating the intrinsic (deterministic) and input (stochastic) components in a similar way as the response to deterministic inputs, for example, in the resonance phenomena described above. A prototypical example is the Ornstein-Uhlenbeck process [81-83] (Appendix B). While the response's expected value coincides with the stationary solution of the noiseless system, the response's variance involves a combination of the model parameters and the variance of the Gaussian white noise input. For example, for the one-dimensional OU process, the variance of the stationary response increases with both the input variance and the cell's time constant. The faster the intrinsic cell's response to constant perturbations (the smaller the cell's time constant), the smaller the magnitude of the response fluctuations. In other words, the variability is controlled by the autonomous transient dynamics, similarly to the resonance phenomena described above. The determination of the dependence of the variability of higher dimensional OU process with the autonomous transient dynamics is more difficult given the complexity of the covariance formulas and it is analytically not possible for noise-driven nonlinear systems including voltage-dependent nonlinearities or conductance-based synaptic inputs.

The goal of this paper is to understand how the intrinsic properties of target cells interact with variable inputs to produce oscillatory and variable responses, and how the response variability is controlled by these intrinsic properties. Similarly to the resonance phenomena described above, we argue that this is mediated by the autonomous transient dynamics, which encodes information about the intrinsic parameters of the target cells under certain constraints (responses to constant inputs), which are possible to determine both computationally and experimentally.

We use piecewise constant (PWC) inputs $I_{\eta}$ with short duration pieces and variable amplitudes that allow for the autonomous transient dynamics to develop. When the set of amplitudes $\eta$ are normally distributed, $I_{\eta}$ is an approximation to Gaussian white noise [84]. We first show that an additive input $I_{\eta}$ with normally distributed amplitudes evoke oscillatory responses in cells whose 
stable equilibria are both foci and nodes, displaying damped oscillations and overshoots in response to constant inputs, respectively. The former captures results described in [82] for Gaussian white noise (see also [85] for two-dimensional linear cells with damped oscillations). We then apply the same input to a nonlinear(piecewise linear, PWL) model [19] mimicking the voltage-dependencies present in neuronal models and to linear models receiving a (multiplicative) conductance-based input and explain how oscillations are nonlinearly amplified or attenuated in these models. Next, we investigate how the response variability depends on the autonomous transient dynamics. All trials for a given protocol have the same set of amplitudes $\eta$, but the order of the constant pieces is different for each trial. Each protocol consists of a subset of all possible order permutations. We compute the peak-and-troughs response profiles $P_{\eta}$ consisting of the set of peaks and troughs of the response evoked by each constant piece and rearrange these profiles according to an increasing order of the input amplitudes. The variability of the $P_{\eta}$ patterns across trials corresponds to the multiple different ways a target cell reacts to the same constant inputs and it is therefore generated by the autonomous transient dynamics. In order to exclude the uncertainty in the input amplitude, we then modify $I_{\eta}$ to have amplitudes distributed according to a deterministic rule and therefore the only uncertainty in the input corresponds to the set of permutations used. The results do not vary significantly, showing that the response variability depends on the multiple ways in which the autonomous transient dynamics are evoked by each constant piece and does not requiere variability in the input. From another perspective, the response variability encodes the autonomous transient dynamics of the target cell. Finally, we show how resonances emerge as we increase the duration of the constant pieces and adding an input frequency to the input that interacts with the autonomous transient dynamics.

\section{Methods}

\subsection{Models}

In this paper we use relatively simple biophysically plausible models describing the subthreshold dynamics of individual neurons subject to both additive and multiplicative inputs.

\subsubsection{Linear model: additive input}

For the individual neurons we use the following linearized biophysical (conductance-based) model $[18,23]$

$$
\begin{aligned}
C \frac{d v}{d t} & =-g_{L} v-g_{1} w+I(t) \\
\tau_{1} \frac{d w}{d t} & =v-w,
\end{aligned}
$$

where $v(\mathrm{mV})$ is the membrane potential relative to the voltage coordinate of the fixed-point (equilibrium potential) of the original model, $w(\mathrm{mV})$ is the recovery (gating) variable relative to the gating variable coordinate of the fixed-point of the original model normalized by the derivative of the corresponding activation curve, $C\left(\mu \mathrm{F} / \mathrm{cm}^{2}\right)$ is the specific membrane capacitance, $g_{L}\left(\mathrm{mS} / \mathrm{cm}^{2}\right)$ is the linearized leak conductance, $g_{1}\left(\mathrm{mS} / \mathrm{cm}^{2}\right)$ is the linearized ionic conductance, $\tau_{1}(\mathrm{~ms})$ is the linearized gating variable time constant and $I(t)\left(\mu \mathrm{A} / \mathrm{cm}^{2}\right)$ is the time-dependent input current. In this paper we consider resonant gating variables ( $g_{1}>0$; providing a negative feedback effect). The linearization process for conductance-based models for single cells has been previously described in $[18,23]$. We refer the reader to these references for details. 


\subsubsection{Piecewise linear (PWL) model: additive input}

To account for nonlinear effects we extend the linear model (1)-(2) to include a piecewise linear function $F_{P W L}[19]$

$$
\begin{aligned}
C \frac{d v}{d t} & =-g_{L} F_{P W L}(v)-g_{1} w+I(t) \\
\tau_{1} \frac{d w}{d t} & =v-w,
\end{aligned}
$$

where

$$
F_{P W L}(v) \begin{cases}v & v<v_{c} \\ v_{c}+g_{c} / g_{L}\left(v-v_{c}\right) & v>v_{c} .\end{cases}
$$

This model has been used to investigate the dynamic mechanisms underlying the nonlinear amplification of the resonant responses to sinusoidal inputs [19] and captures the nonlinear amplification effects of the resonant responses off two-dimensional models with parabolic-like voltage nullclines [20]. Note that the PWL model has linear dynamics for $v<v_{c}$ and therefore the PWL model becomes effectively linear if $v_{c}$ is large enough.

\subsubsection{Conductance-based synaptic input model: multiplicative input}

To account for the effects of conductance-based synaptic inputs we extend the model (3)-(5) to include a synaptic current

$$
\begin{aligned}
C \frac{d v}{d t} & =-g_{L} F_{P W L}(v)-g_{1} w-G_{s y n} S(t)\left(v-E_{s y n}\right) \\
\tau_{1} \frac{d w}{d t} & =v-w
\end{aligned}
$$

where $G_{\text {syn }}\left(\mathrm{mS} / \mathrm{cm}^{2}\right)$ is the maximal synaptic conductance, $E_{\text {syn }}(\mathrm{mV})$ is the synaptic reversal potential and $S(t)$ is the time-dependent synaptic input. We use $E_{s y n}=1$ (excitatory synaptic current).

\subsection{Piecewise constant (PWC) input functions with variable amplitudes}

We use PWC functions with short-duration pieces as a tool to understand how the properties of the transient response of individual cells to constant inputs (autonomous transient dynamics) control the response variability of these cells to time-dependent inputs with variable, abruptly changing amplitudes. White noise and related stochastic inputs (e.g. colored noise) satisfies these last two properties. However, the amplitude changes are too rapid. PWC inputs with variable amplitudes and short durations provide a good balance between input variability and the ability of the autonomous transient dynamics to develop, and therefore they can be linked to the input amplitude that gave rise to them. In addition, PWC inputs with variable amplitudes allow us to use the same set of amplitudes in all trials for each protocol, arranged in different order for each trial, and therefore have a better control of the input-output relationship. PWC functions with normally distributed amplitudes have been used to approximate white noise [84] (see also [86]) and converge to white noise as the duration of all pieces approaches zero. 


\subsubsection{Piecewise constant input functions with normally distributed amplitudes}

We partition the time interval $\left[0, T_{\max }\right]$ into $N$ pieces of equal length $\Delta$, and we define $I_{\eta}(t)=\eta_{k}$ for $t \in\left(t_{k-1}, t_{k}\right)$ for $k=1, \ldots N$. The values of the amplitudes $\left\{\eta_{k}\right\}_{k=1}^{N}$ of the constant pieces are normally distributed (zero mean and variance $D$ ) (Fig. 1, top, see also Fig. 3-a). For each protocol in our study we use multiple different input trial functions $I_{\eta}(t)$ constrained to consist of different (random) permutations of the same set $\left\{\eta_{k}\right\}_{k=1}^{N}$. As part of our analysis we use one specific rearranged version $I_{\eta, \text { step }}$ of $I_{\eta}$ where the linear pieces $\left\{\eta_{k}\right\}_{k=1}^{N}$ are ordered in a monotonically increasing amplitude manner (Fig. 1, middle). We use this as our notion of a reference ("ordered") input in the sense that $I_{\eta, s t e p}$ has the minimum piece-to-piece variability (except possibly for the decreasing order) and it produces the minimum piece-to-piece response variability.

\subsubsection{Piecewise constant input functions with arbitrarily, but deterministically dis- tributed amplitudes}

The choice of normally distributed amplitudes (Fig. 3-a) is motivated by the fact that in the limit of $\Delta \rightarrow 0, I_{\eta}$ approaches white Gaussian noise. In order to decouple the effect of the autonomous transient dynamics evoked by abrupt transitions between constant pieces and the randomness of the signal, we use fully deterministic distributions of amplitudes within some range. More specifically, we use arbitrary order permutations of a number of constant pieces initially arranged in increasing order of amplitudes where the amplitudes are chosen according to deterministic rules. Each protocol consists of a subset of all possible permutations (of the order of constant pieces), which is the only source of uncertainty in the protocol.

We use two types of (deterministic) amplitude distributions for $\left\{\eta_{k}\right\}_{k=1}^{N}$ within the range $\left[\eta_{\min }, \eta_{\max }\right]$ : uniform (Fig. 3-b) and bell-shaped-like (Fig. 3-c). In the uniform distribution the values of $\eta_{k}$ cover the full range $\left[\eta_{\min }, \eta_{\max }\right]$ and satisfy $\eta_{k+1}-\eta_{k}$ is equal for all $k=1, \ldots, N-1$. The "ordered" PWC function $I_{\eta, \text { step }}$ is linear (Fig. 3-b2). The bell-shaped-like distribution was constructed from the random distribution (see the caption in Fig. 3). The "ordered" PWC function $I_{\eta, \text { step }}$ has an inflection point reflecting the higher concentration of input amplitudes around zero (Fig. 3-b3).

\subsection{Output Metrics}

\subsubsection{Impedance (amplitude) profile}

The impedance (amplitude) profile is defined as the magnitude of the ratio of the output (voltage) and input (current) Fourier transforms

$$
Z(f)=\left|\frac{\mathfrak{F}\{v(t)\}}{\mathfrak{F}\{I(t)\}}\right|
$$

where $\mathfrak{F}\{x(t)\}=\int_{0}^{T} d t e^{-2 \pi i f t} x(t)$. In practice, we use the Fast Fourier Transform algorithm (FFT) to compute $\mathfrak{F}\{x(t)\}$. Note that $Z(f)$ is typically used as the complex impedance, a quantity that has amplitude and phase. For simplicity, here we used the notation $Z(f)$ for the impedance amplitude.

\subsubsection{Voltage and impedance (amplitude) envelope profiles}

The upper and lower envelope profiles $V_{E N V}^{+/-}$are curves joining the peaks and troughs of the steady state voltage response as a function of the input frequency $f$. The envelope impedance profile is defined as $[19,20]$

$$
Z_{\mathrm{ENV}}(f)=\frac{V_{E N V}^{+}(f)-V_{E N V}^{-}(f)}{2 A_{\mathrm{in}}},
$$


where $A_{\text {in }}$ is the input amplitude. For linear systems, $Z_{\mathrm{ENV}}(f)$ coincides with $Z(f)$.

\subsubsection{Voltage power spectral density}

In the frequency-domain, we compute the power spectral density (PSD) of the voltage as the absolute value of its Fourier transform $\mathfrak{F}\{v(t)\}$. We will refer to this measure as PSD or $V_{\mathrm{PSD}}$.

\subsubsection{Peaks-and-troughs response profiles}

In each protocol, we compute the cells' voltage response to the multiple trials described before. For each trial we compute the sequence of the response peaks and troughs consisting of the maximum value of the voltage $v$ within the duration of a piece $\eta_{k}$ if $\eta_{k}>\eta_{k-1}$ and the minimum of $v$ within the range of a piece $\eta_{k}$ if $\eta_{k}<\eta_{k-1}$, respectively. We use these peaks-and-troughs patterns or profiles(e.g., Fig. 6-a) to characterize the response patterns and their variability. We note that there are other possible metrics, including the whole $v$ traces and the set of all peaks and troughs present in these traces (e.g., Fig. 6-c to -e). We found the peaks-and-troughs profiles as described above to be simpler and the most useful to compare the responses across trial (permutations).

\subsubsection{Rearranged peaks-and-troughs response profiles}

In order to compare the responses across trials we rearrange the peak-and-troughs response profiles according to the increasing amplitude values of the elements of the set $\left\{\eta_{k}\right\}_{k=1}^{N}$ from which they were evoked. We use the notation $P_{\eta}$ for the resulting rearranged response patterns and $P_{\eta, s t e p}$ for these response profiles produced by $I_{\eta, s t e p}$ (inputs with increasing order of constant piece amplitudes). In this way we compare multiple different ways in which the response to a given constant piece is produced by transitions from pieces with different amplitudes (i.e., different initial conditions with respect to that constant piece). The differences in the peak/troughs values across trials for each piece will be due to the differences in the values of $V$ and the other variables at the arrival time of that piece (initial conditions) and therefore to the differences in the transient dynamics that they evoke.

\subsubsection{Numerical simulations}

We used the modified Euler method (Runge-Kutta, order 2) [87] with step size $\Delta t=0.01 \mathrm{~ms}$. All neural models and metrics, including phase-plane analysis, were implemented by self-developed Matlab routines (The Mathworks, Natick, MA) and are available in https://github.com/BioDatanamics-Lab/ impedance_input_dependent.

\section{Results}

\subsection{Voltage response to piecewise constant inputs with variable amplitudes: sequence of autonomous transient dynamics followed by steady-state re- sponses}

Figs. 1-c1 to -e1 illustrate the voltage response of three types of cells to the same PWC inputs with increasing amplitude (generated by $I_{s t e p}$, see Fig. 1-a, bottom). We use long piece durations here only for explanatory purposes. The response to each linear piece consists of a transient and a steady-state components, which depend on the model parameters. The properties of the transient component in each case (the autonomous transient dynamics) are qualitatively different for the 
three cell types. Passive cells (and cells behaving as passive cells) (Fig. 1-c1) exhibit a monotonic increase towards the steady state. The node cells (cells having a stable node, N-cells) in Fig. 1d1display overshoots and the focus cells (cells having a stable focus, F-cells) in Fig. 1-e1 display damped oscillations before converging to the steady state. We note that not all two-dimensional cells having a stable node or focus show prominent overshoots or damped oscillations.

Figs. 1-c2 to -e2 show the voltage response of the same three cell types to PWC inputs with the same constant pieces as in Figs. 1-c1 to -e1, but the three intermediate pieces are arranged in a different order. The type of autonomous transient dynamics, which are activated a the transition points between input pieces, remains the same as in Figs. 1-c1 to -e1, but the response amplitude of the voltage response pattern increases for the $\mathrm{N}$ - and F-cells as compared to Figs. 1-d1 and -e1. This is due to a combination of factors that involve the differences in the jump sizes between linear pieces and the differences in initial conditions for each linear piece (e.g., Fig. 1-b). The values of the participating variables prior to the transition between linear pieces serve as initial conditions for the new regime. While for 1D system, the response amplitude is bounded by the steady-state for each linear piece regardless of the initial conditions, for 2D systems the amplitude response depend on the initial conditions not only of the main variable $(v)$, but also the recovery variable that remains hidden (e.g., Fig. 1-b).

\subsection{Oscillatory response properties to piecewise constant inputs with arbi- trarily distributed amplitudes}

The $V$ patterns discussed above (Fig. 1, row 2) for a small number of input $\left(I_{\eta}\right)$ partitions (large $\Delta=200$ ) display the fully developed autonomous transient dynamics of the three cell types to step-constant inputs. The extension of these results to the $V$ patterns generated by inputs with a larger, more realistic number of partitions (smaller $\Delta$ ) is not straightforward since the smaller the partition size the less time the cell has to develop the autonomous transient behavior corresponding to each partition. Still, the transient dynamics reflect the model properties and the differences among models.

\subsubsection{Piecewise constant inputs with normally distributed amplitudes and a large enough number of partitions uncovers the oscillatory dynamic properties of the tar- get cells}

Figs. 2 shows the $V$ response patterns to inputs $I_{\eta}$ with a larger number of partitions $(\Delta=5)$. Figs. 2-a1 and -b1 show no apparent oscillatory pattern, while Fig. 2-c1 shows irregular oscillations, which is confirmed by the PSD graph in Fig. 2-c2. The PSD graph in Fig. 2-b2 uncovers the presence of oscillations in the corresponding $V$ pattern (Fig. 2-b1), while the PSD graph in Fig. 2-a2 confirms the absence of oscillations consistent with the 1D dynamics of the cell. Interestingly, not only the F-cell that show damped oscillations in response to step-constant inputs shows oscillatory activity in response to $I_{\eta}$, but also the $\mathrm{N}$-cell that shows overshoots in response to step-constant inputs. This would be in principle not surprising since both cell types show resonance in response to sinusoidal inputs $[18,19]$, but the PSDs for the input patterns $I_{\eta}$ with a smaller number of partitions $(\Delta=200$ as in Fig. 1, row 3) shows no oscillations for the three responses (see insets in Fig. 2, row 2). The arbitrarily (randomly) distributed amplitudes allow the voltage response to explore a wide region of the phase-space and therefore capture information about the vector field governing the transient dynamics. The short duration of the pulses keeps the trajectory ("almost continuously") exploring the vector field by sequentially activating the autonomous transient dynamics, and this "permanent motion" prevents the interference of the regions of the vector field governing the dynamics in close vicinities of the steady state. This mechanism is conceptually similar to the one governing the 
generation of resonance in response to oscillatory inputs in both linear and nonlinear systems [19, 20] where resonance can be observed in the absence of intrinsic oscillatory behavior $[18,19,23]$.

\subsubsection{Uncovering the oscillatory dynamic properties of the target cells requires con- stant pieces with arbitrarily distributed amplitudes, but not amplitude randomness}

Here we decouple the effects of the autonomous transient dynamics (in response to the sequence of input constant pieces) from the pieces' amplitude randomness on the ability of these PWC inputs to uncover the oscillatory (intrinsic) and resonant properties of the receiving cells.

To this end we use fully deterministic distributions of amplitudes within some range as described in Section 2.2.2. This leaves the choice of the subset of all possible permutations (number of trials) for each protocol as the only source of uncertainty in the input signal. Figs. S1 $(\Delta=5)$ and S2 $(\Delta=1)$ show the response patterns to these PWC inputs (uniformly distributed, deterministic amplitudes) for the same parameter values as Fig. 2 (random amplitudes). The $Z$-profiles in these three figures are almost identical. The $V$ PSD profiles in Figs. 2 and $S 1$ are also very similar. The differences between the $V$ PSD profiles in these figures and the ones in Figs. S2 are due to the larger value of the total time used there. Together, these results show that randomness is not needed to uncover the resonant and oscillatory properties of the receiving cells and these oscillatory properties emerge almost exclusively as the result of the sequential and fast activation of the cell's autonomous transient dynamics.

\subsubsection{PWC inputs with arbitrarily distributed amplitudes capture the nonlinear am- plification of the oscillatory responses}

The amplification of the response oscillations to PWC inputs discussed so far was presented in the context of linear systems as the result of changes in the model parameter values (e.g, from N- to Fcells in Figs. 2-, S1-, S2, -b and -c). Because the models used there are linear, the dependence of the response amplitude on the input amplitude $(D)$ is simple. Increasing values of $D$ cause proportional increases in the voltage response amplitude so that the $Z$-profifle remains unchanged.

Here we focus on the nonlinear amplifications produced in (nonlinear) models as the result of increasing values of the input amplitude $D$. To this end we use the piecewise linear (PWL) model (3)-(5), which is a continuous, nonlinear extension of the linear model (1)-(2) where the $V$-nullcline is "broken" (e.g., Figs. 4-b). It was shown in [19] that this type of models display nonlinear amplifications of the voltage response to sinusoidal inputs and capture similar phenomena observed in more realistic nonlinear models, in particular two-dimensional models having parabolic-like $V$-nullclines describing the subthreshold voltage dynamics. [20].

We first introduce the ideas by examining the autonomous transient dynamics of the PWL model in response to a constant input $I$ and then use these results to understand the nonlinear amplification of this PWL model in response to PWC inputs with increasing values of $D$. Figs. 4 -a and $-\mathrm{b}$ show the superimposed phase-plane diagrams for a PWL model (b) and the linear model (a) from which it originates for $I=0$ (solid-red, baseline) and $I=1$ (dashed-red). The $w$-nullclinne is unaffected by changes in $I$. The trajectory (blue), initially at the fixed-point for $I=0$, evolves towards the fixed-point for $I=1$. In both cases (Figs. 4-a and -b) the response exhibits an overshoot. The peaks (inset) occur when the trajectories cross the $V$-nullcline. For low enough values of $I$ (lower than in Figs. 4-a and -b) the trajectory remains within the linear region (the trajectory does not reach the $V$-nullcline's "breaking point" value of $V$ ) and therefore the increasing values of $I$ produce a linear response amplification (no differences between the responses of the linear and PWL model; not shown). The nonlinear amplification is apparent for values of $I$ for which the $v$-nullcline is high enough so the trajectory crosses to the other linear regime. Because the $V$-nullcline's "right piece" has a smaller slope than the "left piece" (the slope it would have if it were linear), the trajectory 
is able to reach larger values of $V$ for the nonlinear system than for the corresponding linear one before turning around, and therefore the peak for the nonlinear system is higher than for the linear system. Nonlinear response amplifications in this type of systems are dependent on the time scale separation between the participating variables. For smaller values of $\tau_{1}$ this nonlinear amplification is reduced and although the system is nonlinear, it behaves quasi-linearly $[19,20]$.

The nonlinear amplification discussed above is particularly stronger for the transient dynamics (initial upstroke) than for the steady-state response, and therefore it is expected to have consequences for the nonlinear responses of nonlinear models to PWC inputs with large enough values of $D$ (Fig. 5). We use a PWC input with a (non-random) uniform distribution of constant piece amplitudes in the range $[0,2]$ multiplied by $D$. For small enough values of $D$ (Fig. 5 -a) the trajectory remains within the linear regime and therefore the responses to the linear and the PWL models are almost identical (blue and red). As $D$ increases, the nonlinear amplification becomes stronger (Figs. 5-b and -c, blue and red). This is accompanied by similar changes in the $Z$ profile (not shown). As discussed above, these mechanisms are dependent on the time scale separation between the participating variables, determined by $\tau_{1}$ and the nonlinear response amplification is reduced for smaller values of $\tau_{1}$ (not shown).

\subsubsection{Oscillation amplification and attenuation: current- versus conductance-based responses to synaptic-like PWC inputs}

The oscillatory dynamics considered above emerge in response to additive PWC current inputs. However, the synaptic inputs received by neurons are multiplicative and conductance-based as described by the model (6)-(7).

From the phase-plane diagram in Fig. 4-c we see that increasing values of $I$ (replacing $S$ in the model) reduces the nonlinearity of the $V$-nullcline (dashed-red) and increases (in absolute value) its slope. Both phenomena oppose the response amplification (blue) and the overshoot becomes much less prominent. The triangular region (bounded by the $V$-axis, the displaced $V$-nullcline (dashedred) and the $w$-nullcline (green)) is reduced in size as compared to the current-based inputs (panel b) and therefore the response is reduced in amplitude. Moreover, because the displaced $V$-nullcline in panel $\mathrm{c}$ is more vertical than the baseline $V$-nullcline, the size of the overshoot in response to constant inputs is reduced and, in this sense, the responses become quasi-1D. As a consequence, the initial portion of the transient responses to abrupt changes in input is reduced in size. This is translates into the oscillatory response to PWC inputs, which is also attenuated and the resonant peak disappears or is significantly reduced (Figs. 5-c, green).

\subsection{Emergence of variability in response to piecewise constant inputs with normally distributed amplitudes}

Here we address the relationship between the transient dynamic properties of individual cells (autonomous transient dynamics) and the variability of their responses to piecewise constant (PWC) input functions with pieces of the same duration and randomly distributed amplitudes. We primarily use Gaussian distributions and a relatively large number of pieces (with a small duration each). This allows us to separate the effects of the autonomous transient dynamics activated by the transition between pieces with variable size-steps from the variability of the frequency input, which is chosen to be in a range much higher than the cell's intrinsic (natural) and resonant frequencies. In the next Section, we show that amplitude randomness is not needed, but only the arbitrary order of the amplitude of the constant pieces. 


\subsubsection{Variability in response to piecewise constant inputs emerges from the tran- sient response properties of the autonomous system to step constant inputs}

The response of cells to PWC inputs consists of a sequence of transient behaviors (initiated immediately after the transition between two constant pieces), reflecting the autonomous transient dynamics in response to a set of initial conditions for the participating variables, followed by an approximation to the steady state for each input piece. The latter and part of the former may be absent if the duration $\Delta$ of each constant piece is not long enough. As discussed above, Figs. 1-c1 to -e1 illustrate three qualitatively different ways in which cells respond to a sequence of increasing step function inputs according to whether they have a stable node $(N, 2 D)$, a stable focus $(F, 2 D)$ or they are passive $(P, 1 D)$. For illustrative purposes, the value of $\Delta$ was chosen to be large enough so as to show both dynamic components of the response to each linear piece. In subsequent simulations, $\Delta$ will be chosen to be much smaller so as to capture realistic situations (as discussed above), and therefore the response will capture only the initial portions of the autonomous transient dynamics.

Passive (1D) cells exhibit a monotonic behavior towards the steady state in response to each input piece. For the parameters chosen, the transient increase is relatively fast (Fig. 1-c1). 2D cells, in addition can display overshoots (Fig. 1-d1, N) and damped oscillations (Fig. 1-e1, F) as they approach the steady state in response to each input piece. The peak amplitudes of $V$ in response to each input piece correspond to the transient component of the response and depend on the initial conditions in that regime (Fig. 1-b), which in turn depend on the values both $V$ and particularly $w$ reach at the end of the previous regime. Because of this sensitivity of the transient responses to initial conditions, an order rearrangement of the constant pieces produces different response patterns (Figs. 1-c2 to -e2). This variability, which is reflected in the the peak and trough values of the "disordered" patterns (c2, d2, e2) as compared to the "ordered" ones (c1, d1, e1) is due to the differences in the initial conditions in each regime as $v$ transitions between pieces with different amplitudes. The variability among patterns generated by different permutations of the set $\left\{\eta_{k}\right\}_{k=1}^{N}$ is inherited from this principle since the initial conditions for a given input piece depends on the "last" values of the participating variables in response to the previous piece. The variability is stronger for the 2D cells ( $d$ and e) than to for the 1D cell (c1) since the sensitivity to initial conditions is weaker for the former than for the latter (the strong dependence on initial conditions is significantly more sensitive for the recovery variable $w$ than for $V$ and 1D cells have no recovery variable).

\subsubsection{The response variability to piecewise constant inputs with normally distributed amplitudes depends on the cell's autonomous transient dynamic complexity}

Fig. 6-a (colored dots) shows the distribution of the peaks-and-troughs patterns $P_{\eta}$ for 100 trials (permutations of a randomly distributed set $\left\{\eta_{k}\right\}_{k=1}^{N}, N=200, \Delta=5$ ) for a passive cell (P), a cell having a node $(\mathrm{N})$ and a cell having a focus $(\mathrm{F})$. The average $\left\langle P_{\eta}\right\rangle$ and the $P_{\eta, \text { step }}$ curves, superimposed to $P_{\eta}$, show that the average across trials and the response to the reference pattern (with minimal variability) are not identical. In this figure they are relatively close.

The variability of $P_{\eta}$ across trials, $\operatorname{Var}\left(P_{\eta}\right)$ is larger for the F-cell than for the $\mathrm{N}$-cell and there seem to be no significant difference between the variability of $\mathrm{N}$ - and $\mathrm{P}$-cells. (The latter two are comparable since they have the same value of $g_{L}$.) However, the qualitative differences between $\mathrm{N}$ and F-cells are accompanied by different strengths in the responses to step-constant inputs (e.g., Fig. 1), and one has to account for these differences in order to isolate the effects of the type of transient dynamics. We note that these parameter values were chosen so that the two cells ( $\mathrm{N}$ and F) have resonance in the same frequency band.

Figs. 6-b1 to -d1 show the variances of $P_{\eta}\left(\operatorname{Var}\left(P_{\eta}\right)\right)$ and Figs. 6-b2 to -d2 show the normalized variances $\left(\operatorname{VarN}\left(P_{\eta}\right)\right)$ computed as these variances divided by the peak of the unforced cells' response to a step-constant input of amplitude 1. Fig. 6-b show that both $\operatorname{Var}\left(P_{\eta}\right)$ and $\operatorname{VarN}\left(P_{\eta}\right)$ are larger for the F-cell than for the $\mathrm{N}$-cell. Fig. 6-c shows that both $\operatorname{Var}\left(P_{\eta}\right)$ and $\operatorname{VarN}\left(P_{\eta}\right)$ are slightly 
larger for the $\mathrm{N}$-cell than for the corresponding P-cell (obtained by making $g_{1}=0$ ). In contrast to this, Fig. 6-d shows that $\operatorname{Var}\left(P_{\eta}\right)$ and $\operatorname{VarN}\left(P_{\eta}\right)$ have different relative values for the F-cell and the corresponding $\mathrm{P}$-cell; $\operatorname{Var}\left(P_{\eta}\right)$ is larger for the $\mathrm{P}$ - than for the $\mathrm{F}$-cell, but $\operatorname{VarN}\left(P_{\eta}\right)$ is larger for the $F$ - than for the P-cell. The values of these quantities in both cases are significantly larger for the F-/P-cells than for the N-/P-cells. Together, these results suggest that under the constraints imposed by the two cells having resonance in the same frequency band, the response of the F-cell is more variable than the response of the $\mathrm{N}$-cell, and the larger variability of the F-/P-cells as compared to the $\mathrm{N}$-/P-cells is due to a smaller value of $g_{L}$, which in turn indicates a stronger amplification of the transient response to step-constant inputs. However, the larger variabilities cannot be attributed to these differences in the response amplitudes since they persist after the variances have been normalized.

A similar result is obtained when one lifts the resonance constraint on the $\mathrm{N}$ - and F-cells. Fig. 7-b shows the effects of changes in $g_{1}$ on $\operatorname{Var}\left(P_{\eta}\right)$ and $\operatorname{VarN}\left(P_{\eta}\right)$ for fixed values of $g_{L}$. As $g_{1}$ increases the transient dynamics of the cell transitions from $\mathrm{P}$ (green) to $\mathrm{N}$ (blue, $f_{\text {res }}=7$ ) to $\mathrm{F}$ (red, $f_{\text {res }}=14$, $f_{\text {nat }}=12.3$ ) (Fig. 7-a). $\operatorname{Var}\left(P_{\eta}\right)$ is slightly larger for the $\mathrm{F}$ - than for the $\mathrm{N}$-cell and larger than these two for the P-cell. In contrast, $\operatorname{VarN}\left(P_{\eta}\right)$ is much larger for the $\mathrm{F}$ - than for the $\mathrm{N}$-cell, and $\operatorname{VarN}\left(P_{\eta}\right)$ for the $\mathrm{P}$-cell is comparable to that for the $\mathrm{N}$-cell.

\subsubsection{The response variability and its own variability depend on the levels of com- plexity of the autonomous transient dynamics}

If the response variability to PWC inputs with randomly distributed amplitudes primarily depends on the receiving cell's autonomous transient dynamics, then one expects the variability to be higher, the faster the response of the individual cells to step-constant inputs. As this response becomes faster, then it is easier for $V$ to reach values further away from the mean and $V$ is more likely to remain away from the mean for a longer time. The parameter $g_{L}$ is the ideal one to test these ideas since it determines the time constant of the $V$ equation. Increasing (decreasing) values of $g_{L}$ cause the response to be slower (faster). Fig. 7-c shows that $\operatorname{Var}\left(P_{\eta}\right)$ is higher for $g_{L}=0.1$ than for $g_{L}=0.2$ and this remains true for various values of $g_{1}$. The dependence of $\operatorname{Var}\left(P_{\eta}\right)$ with $g_{1}$ is more complex. For $g_{L}=0.2, \operatorname{Var}\left(P_{\eta}\right)$ increases with $g_{1}$, while for $g_{L}=0.1, \operatorname{Var}\left(P_{\eta}\right)$ first decreases and then increases with increasing values of $g_{1}$. For $\Delta=1$ these dependences are less well separated (Fig. 7-c2).

For fixed values of $g_{L}$ and $g_{1}$, the time constant $\tau_{1}$ associated to the recovery variable $w$ controls the time separation between the variables $v$ and $w$. The larger $\tau_{1}$ (the slower $w$ ), the faster the autonomous transient response. Because of this stronger sensitivity to initial conditions, the variability is expected to be larger, which is confirmed by Fig. 7-d.

Together, these results and the results of this section show how the variability of the responses to PWC input functions with normally distributed amplitudes are controlled by the transient dynamics of the autonomous responses to piecewise constant inputs. This variability emerges as the input pieces within the same set are permuted for different trials. The differences among the responses to the same piece (piece with the same amplitude) across trials are due to differences in the initial conditions relative to this pieces caused primarily by the varying amplitudes of the preceding pieces. In the controlled situations considered here, the set of pieces for all trials belong to the same set. However, this is not a necessary constraint.

\subsubsection{The peak-and-trough profiles are able to capture the nonlinear properties of the target cells}

In our previous discussion we have used linear models as the receiving cells to the PWC inputs. We have also shown that the presence of certain types of nonlinearities affect not only the vector 
field, but also the effective time constants of the cell, which in turn affect their autonomous transient dynamics. We reasoned that these types of nonlinearities may also affect the variability of the cells' response to PWC inputs. To test these ideas we used the piecewise-linear (PWL) model (3)-(5) where the $v$-nullcline (right hand side of the first equation equal to zero) is broken into two linear pieces at $v=v_{c}$. We chose $v_{c}>0$ to be within the range of values of the $v$ response to the PWC input and $g_{L}>g_{c}$. Effectively, the cell's membrane time constant transitions from $g_{L}^{-1}$ to $g_{c}^{-1}$ at $v=v_{c}$. Therefore one should expect the variability to be larger. Since the PWL model is asymmetric with respect to the equilibrium of the autonomous cell, then one should expect the variability to be asymmetric with respect to the responses mean. Figs. 8 (rows 1 and 2) illustrate these ideas. Figs. 8 (row 3 ) summarize these results and also shows that the variability for the nonlinear models shows a strong dependence on the amplitude of the input constant pieces, while the variability for the linear model is flatter. This is also expected since the larger the input amplitude, the more likely the response to reach values beyond $v_{c}$. However, the presence of nonlinearities in the model does not necessarily guarantees a nonlinear response to external inputs. The latter strongly depends on the time scale separation between the participating variables as it occurs in other types of responses (e.g., to oscillatory inputs) where the mechanisms depend on the cell's autonomous transient dynamics [20]. The stronger the time scale separation (the larger $\tau_{1}$ ), the stronger the nonlinear amplification of the voltage response to time-dependent inputs. Consistent with this, Fig. 8-c1 shows that the peaks-and-troughs profiles for the nonlinear system and $\tau_{1}=10$ is more symmetric and spans a smaller range that the profile for $\tau_{1}=100$ (Fig. 8-b1), while the peaks-and-troughs profiles for the corresponding linear systems are similar (Fig. 8-b2 and -c2). In addition, $\operatorname{Var}\left(P_{\eta}\right)$ is smaller and flatter for $\tau_{1}=10$ (Fig. 8-c1) than for $\tau_{1}=100$ (Fig. 8-c2).

\subsection{The variability in the response to piecewise constant inputs requires constant pieces with arbitrarily distributed amplitudes, but not amplitude ran- domness}

So far we have used PWC input functions with normally distributed amplitudes (Fig. 3-a) motivated by the fact that in the limit of $\Delta \rightarrow 0, I_{\eta}$ approaches white noise. Earlier in the paper we have shown that the ability of PWC inputs to capture the resonant properties of a given cell depends on the multiple ways the receiving cell's autonomous transient dynamics are activated by the different initial conditions resulting from the transition between different linear pieces, while randomness does not play any significant role in this process. Here we extend these ideas to the cells' response variability. To this end we use fully deterministic distributions of amplitudes within some range as described in Section 2.2.2 leaving the choice of the subset of permutations (number of trials) for each protocol as the only source of uncertainty in the input signal.

Figs. 9, S3 and S4 show that for the three types of deterministic distributions presented in Fig. 3, the PWC inputs uncover the oscillatory properties of receiving cells (top panels) and show the same type of variability as the responses to the normally distributed PWC inputs discussed above (bottom panels). The differences are in the details. The main observed differences (by inspection) are in the responses to the $\mathrm{P}$ cells (panels a2) where the $\operatorname{Var} P_{\eta}$ profiles are more dense for the uniform than for the bell-shaped-like PWCs and differences in the values of $D$ for the latter are reflected in the peaks-and-troughs distributions in the $\operatorname{Var} P_{\eta}$ profiles. A more detailed analysis is beyond the scope of this paper. 


\subsection{The activation of the autonomous transient dynamics by the piecewise constant inputs with small $\Delta$ evokes the steady-state oscillatory properties of the receiving cells, and not their transient oscillatory properties}

Along the previous sections we have shown a number examples where both the impedance amplitude $Z(f)$ and the voltage PSD profiles of cells receiving PWC inputs with small durations $\Delta$ exhibit resonance, independently of whether the corresponding autonomous systems are a node (no intrinsic oscillations) or a focus (intrinsic damped oscillations). The impedance amplitude profile $Z(f)$ of a system captures its steady state response to oscillatory inputs. For linear systems, it is the magnitude of the complex-valued coefficient of the particular solution to the system (see Appendix A.2) forced by a sinusoidal function. In this calculation, the transient component of the solution to the forced system (the solution to the corresponding homogeneous system) is ignored. The resonance frequency $f_{\text {res }}$, the peak frequency of $Z(f)$, is different from the natural frequency $f_{\text {nat }}$ (the frequency of the transient damped oscillations). The latter is computed from the eigenvalues for the homogeneous system (see Appendices A.1 and A.2, and also [18, 23]), which controls the autonomous transient dynamics.

On the other hand, the dynamic mechanisms of generation of resonance involve the autonomous transient dynamics as uncovered by using dynamical systems tools $[19,20]$. Most significantly, the interplay of the input frequency (whose inverse is a measure of the input time scale) and the cell's intrinsic time scale determines the direction of motion of the response limit cycle trajectory in the phase-space. For the resonance frequency, this interaction of time scales produces the limit cycle trajectory with the maximal amplitude in the $v$ direction. This mechanism does not rely on the cell's eigenvalues and eigenvectors (autonomous steady-state dynamics) and it underlies the resonant responses of both $\mathrm{N}$ - and $\mathrm{F}$-cells.

Because white noise has a constant PSD, both the $Z$ and the $V$ PSD profiles have the same frequency-dependent properties. This extends to PWC inputs with small enough values of $\Delta$ (e.g., Figs. 2a2 to -c2, Figs. 9- and S3- to S4-a1 to -b1), but not necessarily for large values of $\Delta$ (e.g., Figs. 2a2 to -c2, insets). Fig. 9-b1 (see also Figs. S3- to S4-b1) shows that both the $Z$ and the $V$ PSD profiles peak at $f_{\text {res }}$ when $f_{\text {nat }}=0$ (the receiving cell is a node). For these parameter values there is a clear separation between the cell's response to the oscillatory input and the autonomous intrinsic dynamics (captured by $f_{\text {nat }}$ ). In Fig. 9-c1 (see also Figs. S3-c1 to S4-c1) the $V$ PSD profile seems to be superimposed to the $Z$ profile, but because $f_{\text {res }}$ and $f_{n a t}$ are very close, it is not clear whether the $V$ PSD profile peaks at $f_{\text {res }}, f_{\text {nat }}$ or in between. For the parameter values in Fig. 10-a1 the separation between $f_{\text {res }}$ and $f_{\text {nat }}$ is bigger and the $V$ PSD profile clearly peaks around $f_{\text {res }}$ and far away from $f_{\text {nat }}$ for $\Delta=1$.

In contrast, Figs. 10-b show more complex responses for $\Delta=50$. The duration of the constant pieces $\Delta$ determines an input time scale that interacts with the cell's intrinsic time scale to produce the $V$ response. This is true for all values of $\Delta$, but for small values of $\Delta$ the corresponding frequencies $(1000 / \Delta)$ are very large, away from the resonant frequency band, and the response time to each constant input piece very small. As $\Delta$ increases, the interaction between time scales is stronger and felt for the lower frequencies. For example, in Figs. 10-b for $\Delta=50$, the $V$ PSDs show resonances occurring at multiples of $1000 / \Delta=20$. The shape of these resonant patterns reflects the properties of the autonomous transient dynamics rather than the properties of the corresponding $Z$ profiles. In Figs. 10-b1, the sequence of PSD peaks decreases, while in Figs. 10-b2, the sequence of PSD peaks increases and the maximum of these peaks (save the maximum at $f=0$ ) occurs roughly at $f_{\text {nat }}$. This reflects the stronger oscillatory responses evoked by each constant piece due to their autonomous transient dynamics.

In summary, the autonomous transient dynamics plays a role in shaping the $V$ response PSD patterns, but these patterns transition from reflecting the stationary properties of the $V$ response to oscillatory inputs, captured by the $Z$ profile, to the intrinsic properties of the receiving cell as $\Delta$ increases. 


\section{Discussion}

Neuronal systems are subject to fluctuations either intrinsically or externally [25-34, 88, 89], which have been modeled as random Gaussian white or colored noise [42]. Cells subject to variable inputs have been shown to exhibit a number of non-expected behaviors (see Introduction for more details and references), including oscillatory responses and resonances, and they also exhibit variability across trials. These phenomena result from the dynamic, often non-trivial interplay of the cell's intrinsic properties and the properties of the noise. While variable inputs are ubiquitous, in particular in neuronal systems [26, 42, 90], several aspects of the dynamic mechanisms governing these interactions are not well understood. In particular, it is not well understood what aspects of the cell's intrinsic dynamics play a role in each of these interactions, what properties of the variability are necessary, if at all, and how the two are integrated to produce these behaviors. Linear systems receiving Gaussian white noise can be solved analytically [81-83], but, in general, the resulting formulas provide limited mechanistic information. Nonlinear systems are not amenable to analytical solutions.

In previous work we showed that the cell's autonomous transient dynamics (the cell's transient response to constant inputs, which is uncovered by the response to abrupt changes between two constant inputs) play a significant role in the mechanistic explanation of the generation of resonance in linear and non-linear systems in response to oscillatory inputs $[19,20]$. Even for linear systems for which the impedance amplitude and phase profiles can be computed analytically, the resulting formulas do not explain why and under what circumstances resonance emerges in systems that do not display intrinsic oscillations (i.e., systems having only real eigenvalues). We developed a dynamical systems approach to show that the cell's response to oscillatory inputs can be thought of as a continuous sequence of transient responses to constant inputs equal to the value of the input at a given time. The response trajectory for each input frequency tracks the cyclic motion of the voltage nullcline in the extended phase-plane diagram (technically, the projection of a surface in the three-dimensional space) and this evolution is governed by the the properties of the autonomous transient dynamics. The resonant frequency corresponds to the optimal balance between the effective time scale at which the system transiently reacts to constant inputs and the input frequency. This could be captured by approximating the sinusoidal inputs by piecewise constant (PWC) functions with short-duration pieces and computing the sequence of (property joined) response trajectories to these PWC inputs. Because in general these trajectories do not reach a close enough vicinity of the equilibrium for each constant piece, knowledge of the the steady-state properties of the unforced target cell (eigenvalues and eigenvectors) does not inform the generation of resonance. We reasoned that similar ideas could shed light on the issues raised in the previous paragraph.

In this paper we set out to investigate the mechanisms of generation of oscillations and variability in response to PWC inputs $I_{\eta}$ with short-duration constant pieces and variable amplitudes. For each constant piece, the autonomous transient dynamics are able to develop, but the response does not reach a close vicinity of the corresponding steady-state. For randomly distributed amplitudes $\eta$, the PWC inputs provide an approximation to Gaussian white noise [84]. However, although our results have implications for systems subject to random Gaussian noise, we are not making precise statements about the transition from the responses to the deterministic PWC inputs to the responses to stochastic Gaussian noise inputs as the duration of the constant pieces approaches zero. This requires more research and is beyond the scope of this paper.

We showed that oscillatory behavior can be generated in response to additive PWC inputs $I_{\eta}$ in both linear and nonlinear systems, independently of whether the stable equilibria of the unperturbed systems are foci or nodes. These results persisted when the amplitudes of the constant pieces were not randomly distributed, but chosen following a deterministic rule. For linear systems, this captures earlier results described in [82] for Gaussian white noise (see also [85] for two-dimensional linear cells with foci). However, the dynamic mechanisms of generation of oscillations and their dependence on the autonomous transient dynamics, in particular for cells having a node were not known. 
To understand the effects of the nonlinearities on the oscillatory response we used a piecewise linear model (PWL) developed in [19] that mimics the voltage-dependences present in neuronal models in the voltage nullclines (for the current-balance equation). We showed that the oscillatory response is amplified by the nonlinearities. This nonlinear amplification is consistent with previous work on resonance (for sinusoidal inputs) [19] and can be also explained in terms of the interaction between autonomous transient dynamics and the input properties. The PWL model has two regimes, the linear regime, in the vicinity of the fixed-point, and the nonlinear regime for voltage values higher than the "breaking point" determining the boundary between the two PWL input pieces (see Fig. 4-b). The nonlinear regime is in itself linear with respect to a virtual fixed-point, which is different from the actual fixed-point (the origin in Fig. 4-b). For each constant input piece for which the response trajectory crosses to the nonlinear regime, this trajectory is able to reach larger voltage values as compared to the linear model for the same input. This is because the slope of the voltage nullcline in the nonlinear regime is larger (less negative) than the slope in the linear regime, and therefore the trajectory for transient response to the corresponding constant input is able to jump to higher values as compared to the linear regime. This clearly depends on the properties of the PWL model, in particular on the slope of the "broken" linear piece, which we chose to be larger (less negative) than the linear piece in the linear regime (see Fig. 4-b). Other slopes may lead to attenuation of the response. Following similar ideas, we showed that the response to multiplicative conductancebased synaptic inputs are attenuated as compared to the underlying model with additive noise. In this case, the "broken" piece is more negative than the linear one (see Fig. 4-c). This has mechanistic implications for the experimental results discussed in [91], comparing the responses of currentand conductance-based synaptic fluctuations. A more detail explanation requires the use of highfrequency Poisson-distributed synaptic-like inputs. We analyze this in more detail in a companion paper.

The effect of the autonomous transient dynamics on the response variability to $I_{\eta}$ is already apparent in the simple illustrative example in Figs. 1-c to -e. The response variability emerges as the result of the transition between regimes corresponding to different constant pieces. The variability is minimal for the response to $I_{\eta, s t e p}$ (Figs. 1-c1 to -e1) where the constant pieces are arranged by increasing amplitude and the variability increases as the constant pieces are permuted (Figs. 1-c2 to -e2) since the transient response for each constant piece regime depends on the initial conditions for that regime. These, in turn, depend on the value of the participating variables at the end of the previous regime, which is different for different trials (different permutations of the order of the constant pieces). To clarify these ideas we designed protocols where the set of amplitudes $\eta$ was the same for all trials and each trial used a different permutation of this set. We then rearranged the response peak-and-trough profiles $P_{\eta}$ in increasing order of input amplitudes $\eta$. In this way we were able to compare the different responses to the same input amplitude across trials. We used both randomly distributed amplitudes and deterministic distribution of amplitudes. For each input amplitude $\eta_{k}$, the variability in $P_{\eta_{k}}$ across trials was the result of the multiple different ways the target cell reacts to the input $\eta_{k}$. Again, this is due to the differences in the initial conditions for that regime, which in turn depend on the values of the participating variables at the end of the previous regime. This variability was strongly affected by the properties of the target cells and their ability to produce overshoots and damped oscillations in response to abrupt changes in constant inputs, which in turn depends on the model parameters, which constrain these responses. This interpretation is strengthened by the fact that the variability patterns remained qualitatively the same when we relaxed the requirement that the amplitudes are randomly distributed and we used permutations of the same deterministic distribution for each protocol. Although it was not the full overshoot or the damped oscillations what affected the response, but rather the initial portion to these responses, the particular dependence on the model parameters remain. In other words, cells that exhibits stronger autonomous transient dynamics show more variability. In this sense, this is consistent with the results for one-dimensional OU processes and it is intuitive for higher-dimensional OU processes, however it is not directly clear from the complex covariance formulas. For nonlinear proceses, analytic computations are not pos- 
sible and therefore the method we developed allows to make predictions based on the knowledge of the autonomous transient dynamics and the structure of the input. Our results further predict that the variability would be reduced if the input functions involve gradual rather than abrupt transitions.

These predictions and the predictions about the oscillatory properties of cells in response to PWC inputs could be tested experimentally in vitro using the dynamics clamp technique [92,93] or in vivo using optogenetic tools [94-96]. In the first case, variability in response to PWC inputs is expected to be well correlated with an appropriate metrics for the autonomous intrinsic dynamics measured in response to constant inputs. Obtaining these correlations in the second case could be more challenging, but still possibly by using the appropriate tools to measure the response membrane potentials.

Another, more involved experimental test or application of the ideas presented in this paper is the presentation of complex odors consisting of sequences of different permutations of a number of "basic odors" [11,17]. Variability in the responses is expected across trials and an increase in the variability patterns is expected as the "intensity" of these odors increase. However, odor representations in the olfactory research is a network phenomena and therefore additional research is needed to extend our results to include network effects.

Our results have also implications for the understanding of the emergence of variability in neuronal systems [97] and dynamical systems in general, and in the absence of noise. In the simple systems we used here, variability emerges as the result of the interaction between the system's autonomous transient dynamics and a set of deterministic inputs where the only source of uncertainty is the choice of the subset of all possible PWC functions with the same set of constant piece amplitudes. Key for this variability to emerge are the abrupt transitions between constant piece regimes and the sensitivity to initial conditions of the autonomous transient dynamics. In higher order networks, this can be evoked by the network internal dynamics without the need of external inputs. Therefore our results have implications for the encoding of information in general and for these resulting from expansions or quenching of variability $[98,99]$. More research is needed to establish in these networks how variability is affected by the node and connectivity parameters. Finally, if variability encodes the autonomous transient dynamics of the target cells, then decoding methods should be able to infer these dynamics. These also requires more research.

\section{Acknowledgments}

This work was partially supported by the National Science Foundation grant DMS-1608077 (HGR). 
bioRxiv preprint doi: https://doi.org/10.1101/2021.06.14.448371; this version posted June 15, 2021. The copyright holder for this preprint (which was not certified by peer review) is the author/funder, who has granted bioRxiv a license to display the preprint in perpetuity. It is made available under aCC-BY 4.0 International license.

\section{Figures}


a
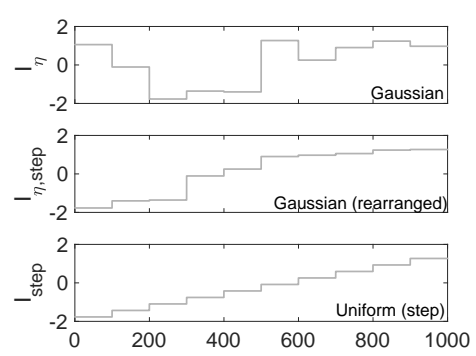

Passive

c1

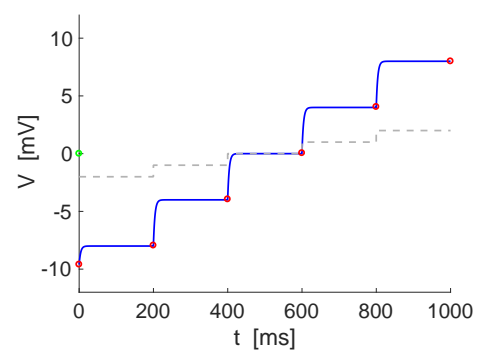

c2

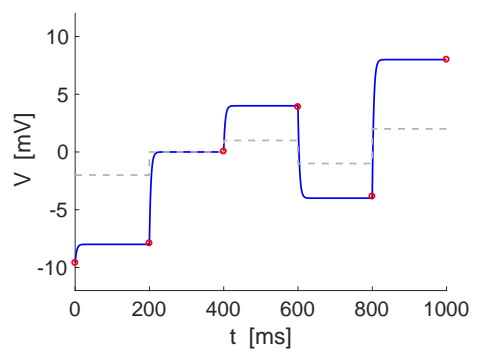

b1

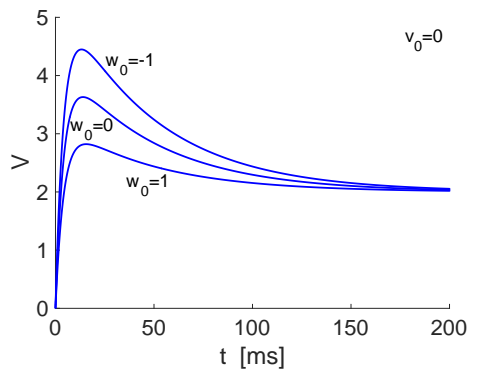

Node

d1

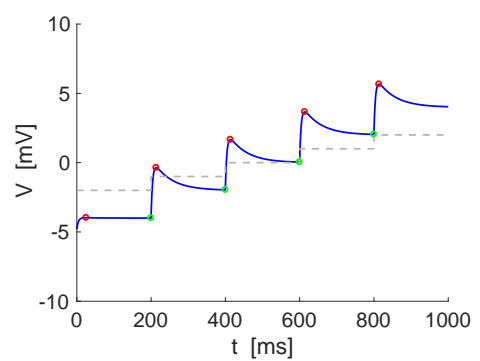

d2

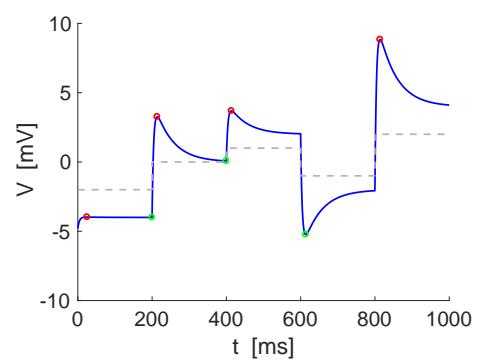

b2

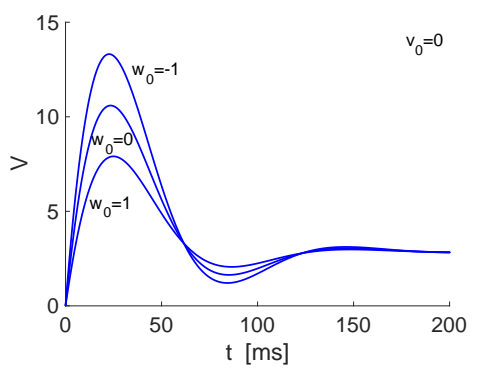

Focus

e1

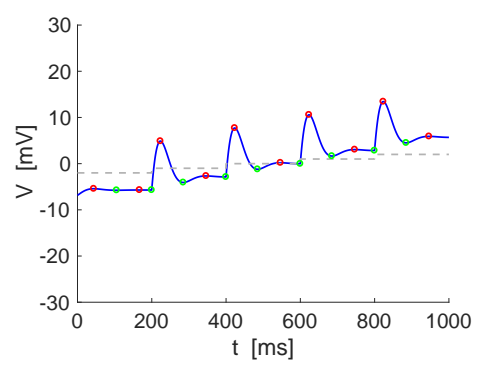

e2

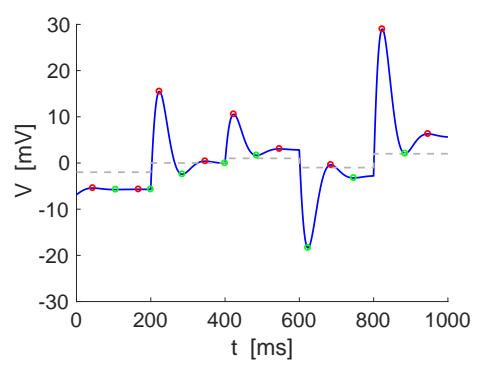

Figure 1: Representative responses of linear systems to piecewise constant inputs with normally distributed amplitudes. a. Representative examples of piecewise constant inputs $(N=10, \Delta=100)$. Top: the values of the constant pieces of $I_{\eta}$ are normally distributed (mean zero and variance one). Middle: the values of the constant pieces of $I_{\eta, s t e p}$ are as in the top panel, but rearranged in increasing order. Botton: the values of the constant pieces of $I_{\text {step }}$ are such that the steps are equal with $\eta_{1}$ and $\eta_{N}$ taken from $I_{\eta, s t e p}$ (bottom). In other words, $I_{\eta, s t e p}$ and $I_{\text {step }}$ change in between the same values $\left(\eta_{1}\right.$ and $\eta_{N}$ ) across trials. b. Dependence of the overshoot (b1) and first oscillation (b2) peaks with the initial value $w(0)=w_{0}$ for the recovery variable $w$ in response to the same constant input $I_{a p p}=1$ and $v(0)=0$. Changes in the initial value of $v$ produces only minor changes in the response patterns. $\mathbf{c}$ to e. Response of cells with three qualitatively different types of autonomous dynamics to piecewise constant inputs $(N=5, \Delta=200)$. Red and green dots indicate peaks and troughs, respectively. Row 2. $I_{\text {step }}$ ordered by increasing values of $\left\{\eta_{k}\right\}_{k=1}^{5}:(-2,-1,0,1,2)$. Row 3. $I_{\eta}$ using the values of $\left\{\eta_{k}\right\}_{k=1}^{5}$ : $(-2,-0,1,-1,2)$ as in row 2, but with a different (non-monotonic) distribution. c. Passive cell. We used the following parameter values: $C=1, g_{L}=0.25$. Red dots indicate the boundaries between constant pieces (there are no peaks and troughs). d. 2D linear system exhibiting an overshoot in response to step-constant inputs. We used the following parameter values: $C=1, g_{L}=0.25, g_{1}=0.25, \tau_{1}=100$. e. $2 \mathrm{D}$ linear system exhibiting damped oscillations in response to step-constant inputs. We used the following parameter values: $C=1, g_{L}=0.05, g_{1}=0.3, \tau_{1}=100$. 

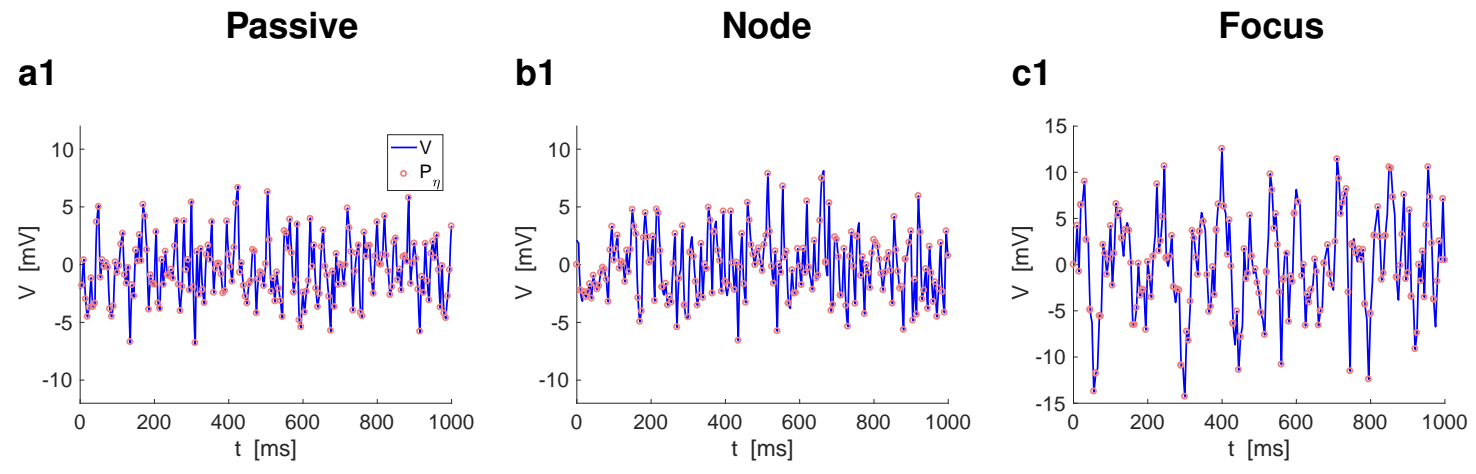

a2

b2

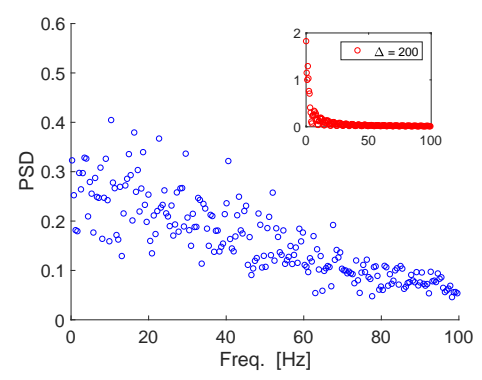

a3

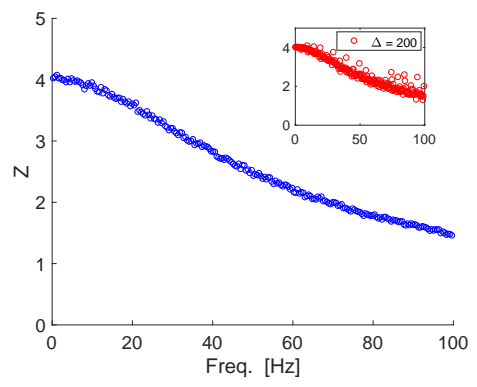

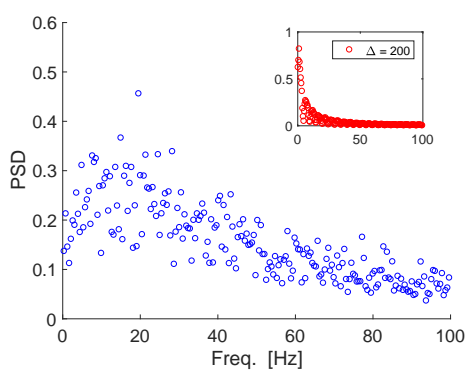

b3

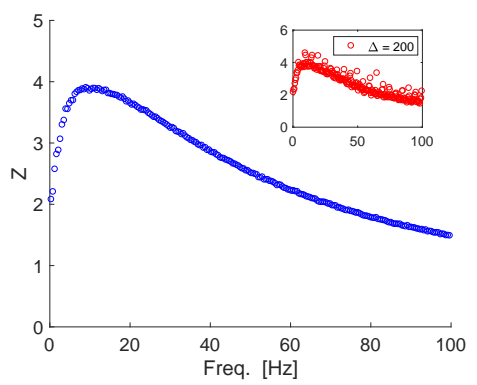

c2

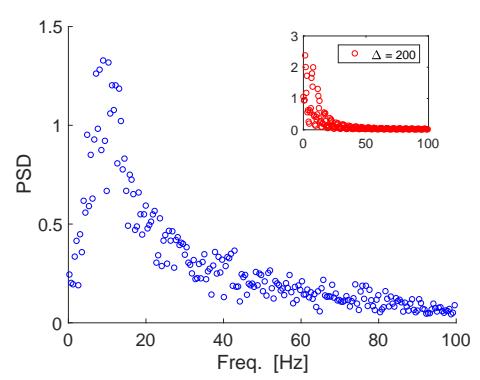

c3

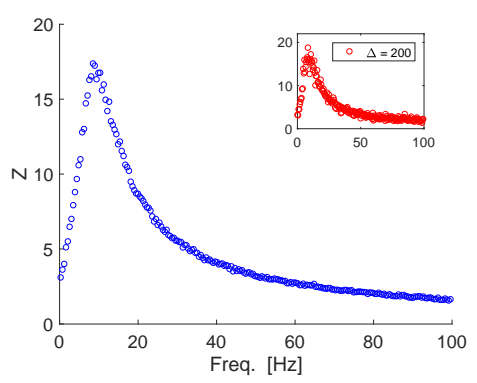

Figure 2: Piecewise constant inputs with normally distributed amplitudes capture the transient dynamics of the target cells. The piecewise constant inputs $I_{\eta}$ have $\Delta=5$ (total time $=10000 \mathrm{~ms}, \mathrm{~N}=2000$ ). The insets have $\Delta=200$ (total time $=10000 \mathrm{~ms}, \mathrm{~N}=50$ ). The parameter values are the same as in Fig. 1. Row 1. Representative $V$ traces (only $1000 \mathrm{~ms}$ are shown). The coral dots indicate peaks-and-troughs patterns computed as the maximum (minimum) of the $V$ response if $\eta_{k}>\eta_{k-1}\left(\eta_{k}<\eta_{k-1}\right)$. Row 2. Power spectra density (PSD) profiles for the sample $V$ trace. Row 3. Impedance amplitude $(Z)$ profiles for the sample $V$ trace. a. Passive cell $\left(f_{\text {nat }}=f_{\text {res }}=0\right)$. We used the following paramete.r values: $C=1, g_{L}=0.25$. b. $2 \mathrm{D}$ linear system exhibiting an overshoot in response to step-constant inputs ( $\left(f_{\text {nat }}=0, f_{\text {res }} \sim 9 H z\right)$. We used the following parameter values: $C=1, g_{L}=0.25, g_{1}=0.25, \tau_{1}=100$. c. $2 \mathrm{D}$ linear system exhibiting damped oscillations in response to step-constant inputs $\left(f_{\text {nat }} \sim f_{\text {res }} \sim 8 H z\right)$. We used the following parameter values: $C=1$, $g_{L}=0.05, g_{1}=0.3, \tau_{1}=100$ 
a

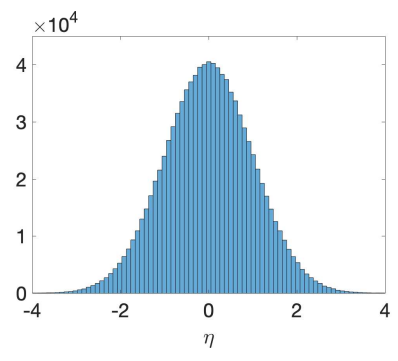

c1

$D=1.5$

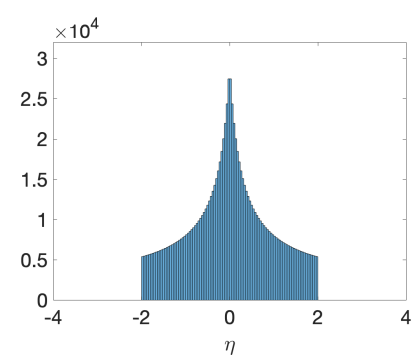

b1

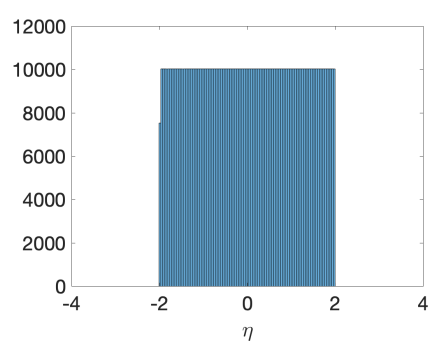

c2

$D=1$

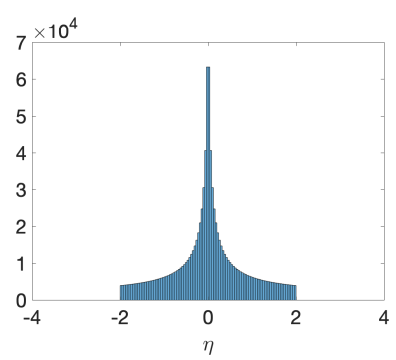

b2

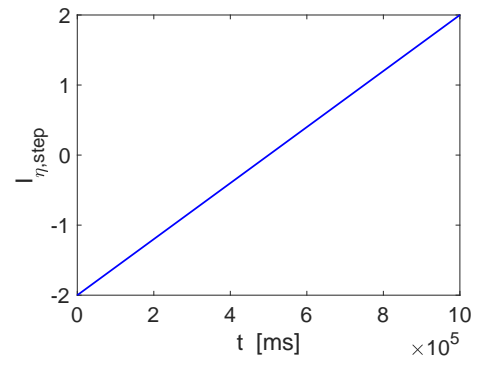

c3

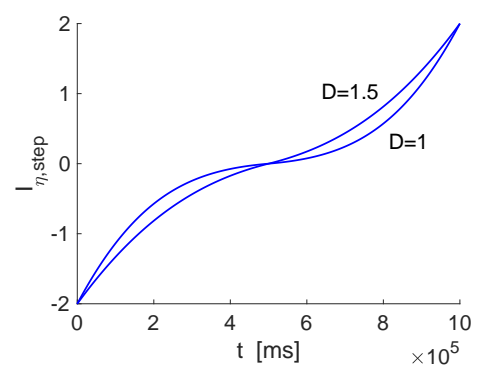

Figure 3: Histograms for representative distribution of the piecewise constant input amplitudes $(\eta)$ of $I_{\eta}$. To construct $I_{\eta}(t)$ we partition the time interval $\left[0, T_{\max }\right]$ into $N$ pieces of equal length $\Delta$, and we define $I_{\eta}(t)=\eta_{k}$ for $t \in\left(t_{k-1}, t_{k}\right)$ for $k=1, \ldots N$. The values of the amplitudes $\left\{\eta_{k}\right\}_{k=1}^{N}$ of the constant pieces are selected by following different rules (panels a to c). Each realization of $I_{\eta}(t)$ corresponds to a permutation of the order of the set $\left\{\eta_{k}\right\}_{k=1}^{N}$. $I_{\eta, s t e p}(t)$ is the special case where $\eta_{k}$ are organized in a non-decreasing order (used for reference). In all cases, $\Delta=1$ with a total time = $1000000 \mathrm{~ms}$. a. Random normal distribution with mean zero and variance one. b. Uniform (deterministic) distribution in the $\eta$ interval $[-2,2] . I_{\eta, s t e p}(t)$ consist of the $\left\{\eta_{k}\right\}_{k=1}^{N}$ organized in an increasing order of amplitudes with c constant amplitude difference between adjacent pieces . c. Bell-shaped-like (deterministic) distribution in the $\eta$ interval $\left[\eta_{\min }=-2, \eta_{\max }=2\right]$. They were computed by (i) generating a uniform distribution in the $\eta_{a u x}$ interval $\left[\eta_{\min }, \eta_{\max }\right]$, (ii) generating a cumulative distribution function (CDF) over $\eta_{a u x}$ of a normal distribution with mean zero and variance $D$, (iii) computing $A m p_{a u x}$ by flipping over (left to right) the left half the sequence of the CDF and multiplying it by 2 so to the resulting $A m p_{\text {aux }}$ lies in the interval [0,1], (iv) Computing $A m p_{\text {int }}=A m p_{a u x} *\left(\eta_{\max }-\eta_{\min }\right) /\left(2 * \Sigma A m p_{a u x}\right)$. The right hand is computed by making it symmetric to the left hand over the ordinates axis. The ordered sequence $\left\{\eta_{k}\right\}_{k=1}^{N}$ is computed from these amplitudes. c1. $D=1.5$. c2. $D=1$. 
a

LIN Current-based

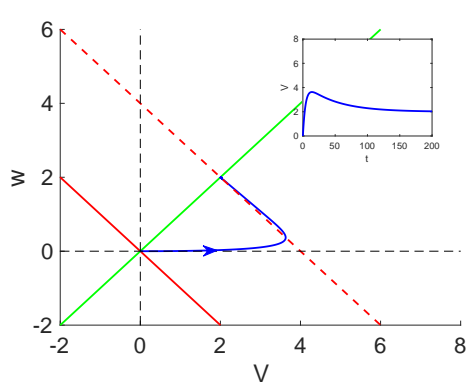

b

PWL Current-based

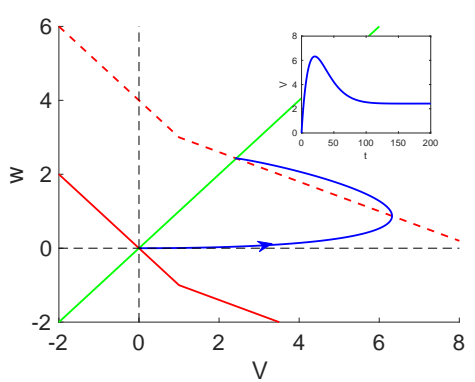

C

\section{PWL} Conductance-based

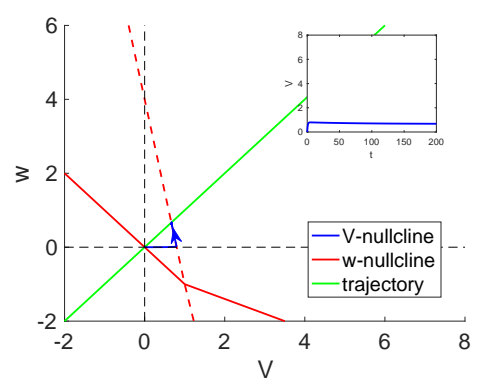

Figure 4: Nonlinear transient response amplifications and attenuations in current- vs. conductance-based inputs. Phase-plane diagrams for $I=1$. The solid-red curve represents the $V$-nullcline for $I=0$, The dashed-red curve represents the $V$-nullcline for $I=1$, the solid-green curve represents the $w$-nullcline for $I=0$, and the solid-curve represents the trajectory initially at $(0,0)$ (the fixed-point for $I=0$ ), converging to the fixed-point for $I=1$. The insets show the $V$ traces. The $2 \mathrm{D}$ linear system exhibits and overshoot in response to step-constant inputs and resonance in response to oscillatory inputs $[18,19,23]$. a. Linear (LIN) model described by eqs. (1)-(2). b. Current-based piecewise linear (PWL) model described by eqs. (3)-(5). c. Conductance-based piecewise linear (PWL) model described by eqs. (6)-(7) and (5) with $S$ substituted by $I$. We used the following parameter values: $C=1, g_{L}=0.25, g_{1}=0.25, \tau_{1}=100$ (same as in Figs. 1 and 2-b), $v_{c}=1$ and $g_{c}=0.1$.

a

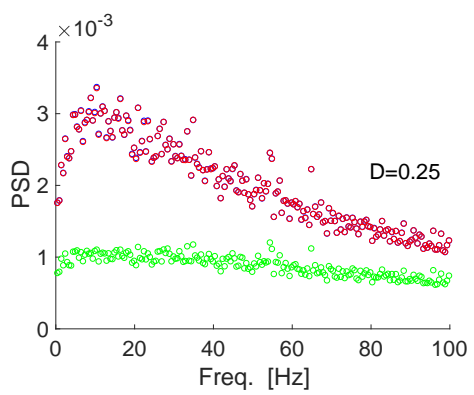

b

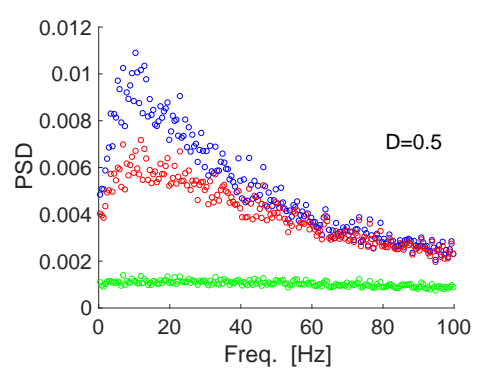

C

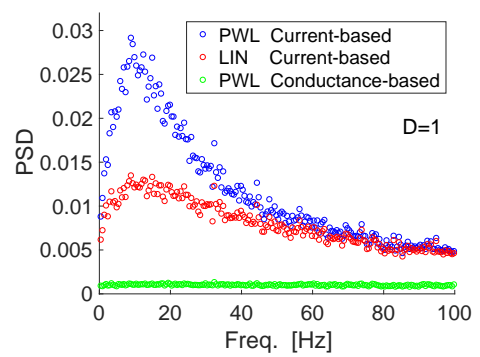

Figure 5: Oscillatory responses for current- vs. conductance-based piecewise constant inputs with uniformly (non-randomly) distributed amplitudes. The piecewise constant inputs $I_{\eta}$ have $\Delta=1$ (total time $=1000000 \mathrm{~ms}, \mathrm{~N}=$ 1000000). The constant pieces' amplitudes are uniformly and deterministically distributed in the range $[0,2]$. The $2 \mathrm{D}$ linear system exhibits and overshoot in response to step-constant inputs and resonance in response to oscillatory inputs. Parameter values are as in Fig. 5 (the parameter values for the linear systems are the same as in Figs. 1 and 2-b). Power spectra density (PSD) profiles for a sample $V$ trace. The piecewise constant inputs $I_{\eta}$ (total time $=1000000 \mathrm{~ms}, \mathrm{~N}=1000000$ ) have different values of $D$. a. $D=0.25$. b. $D=0.5$. c. $D=1$. We used the following parameter values: $C=1, g_{L}=0.25, g_{1}=0.25$, $\tau_{1}=100, v_{c}=1$ and $g_{c}=0.1$. 
a1

Passive

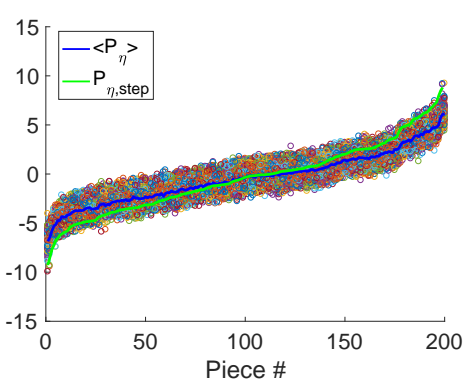

Node/Focus

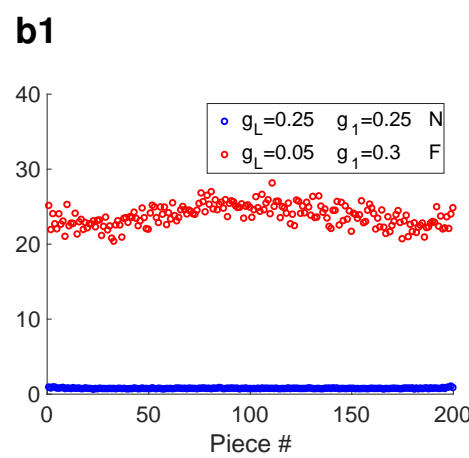

b2

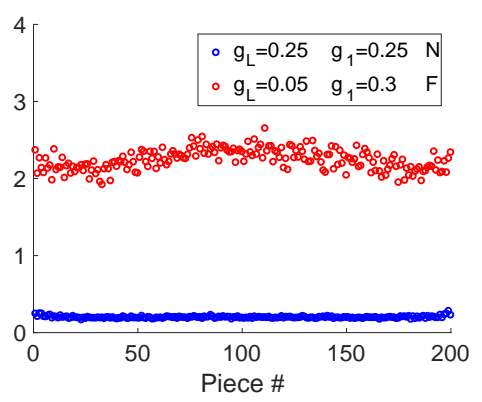

a2

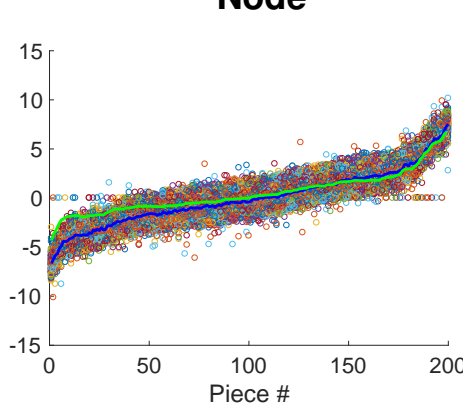

Node/Passive

c1

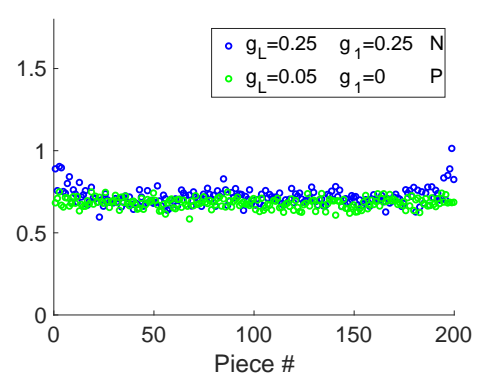

c2

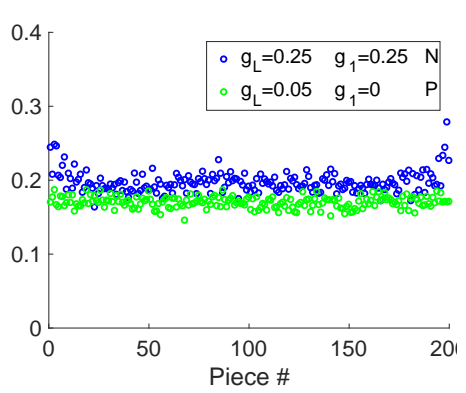

a3

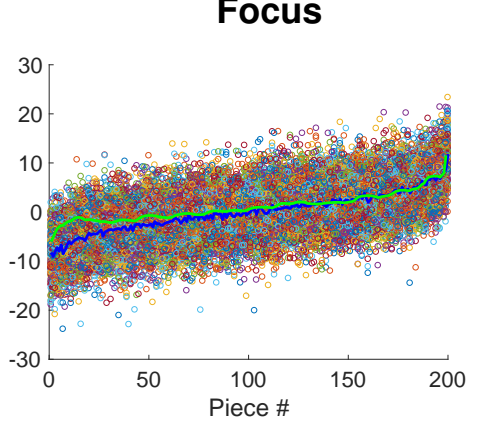

Focus/Passive

d1

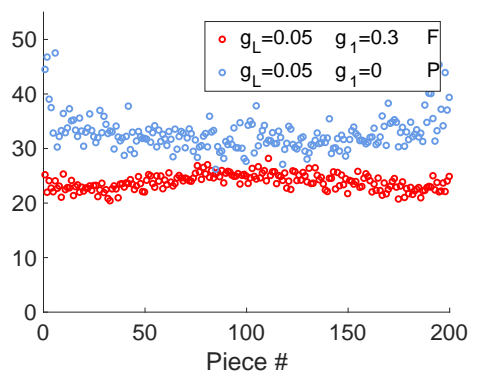

d2

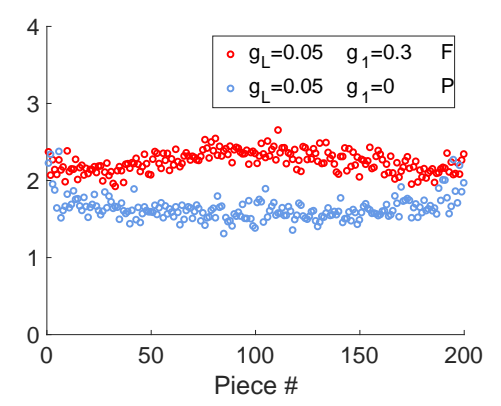

Figure 6: Variability (across trials) of the responses of linear systems to piecewise constant inputs with normally distributed amplitudes. Trials consist of different permutations of the same set of constant pieces $\left\{\eta_{k}\right\}_{k=1}^{N}$. The piecewise constant inputs $I_{\eta}$ have $\Delta=5, N=200(\mathrm{a}, \mathrm{b})$ and $N=2000$ (a). The parameter values are the same as in Fig. 1 . The color dots indicate the peaks-and-troughs patterns for all trials reorganized so that the corresponding values of $\eta_{k}$ from which they originate are ordered in a monotonically increasing manner. All dots for a given piece correspond to the same value of $\eta_{k}$. $\left\langle P_{\eta}\right\rangle$ (blue) is the mean value of the reordered peaks-and-troughs patterns for each linear piece. $\left\langle P_{\eta, s t e p}\right\rangle$ (green) is the peaks-and-troughs pattern corresponding to the (ordered) input function $I_{\eta, s t e p}$. b1 to d1. Variance (b1 to d1) of the peaks-and-troughs patterns in panel a. b1. Comparison between cells having a node and a focus. c1. Comparison between a cell having a node and the corresponding passive cell. d1. Comparison between a cell having a focus and the corresponding passive cell. b2 to d2. Normalized variance (b2 to d2) computed as the variance (b1 to d1) divided by the peak of the unforced cells' response to a step-constant input of amplitude 1. b2. Comparison between cells having a node and a focus. c2. Comparison between a cell having a node and the corresponding passive cell. d2. Comparison between a cell having a focus and the corresponding passive cell. We used the following parameter values: (i) $C=1, g_{L}=0.25$, $g_{1}=0.25, \tau_{1}=100$ for the node, and (ii) $C=1, g_{L}=0.05, g_{1}=0.3, \tau_{1}=100$ for the focus. 
bioRxiv preprint doi: https://doi.org/10.1101/2021.06.14.448371; this version posted June 15, 2021. The copyright holder for this preprint (which was not certified by peer review) is the author/funder, who has granted bioRxiv a license to display the preprint in perpetuity. It is made available under aCC-BY 4.0 International license.

a

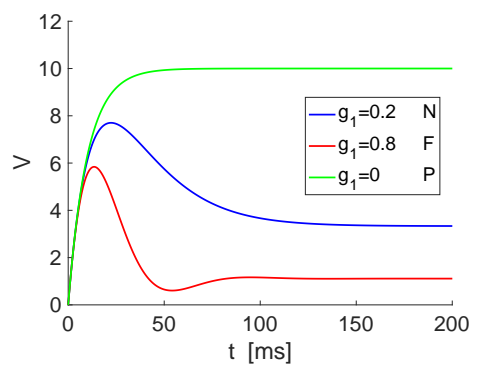

c1

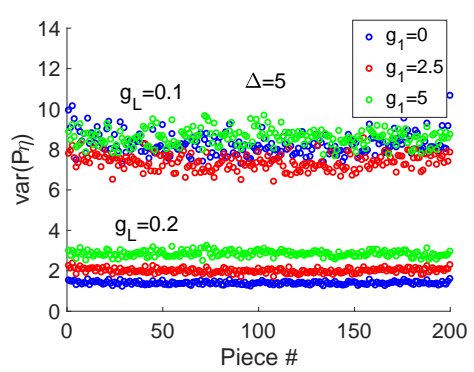

b1

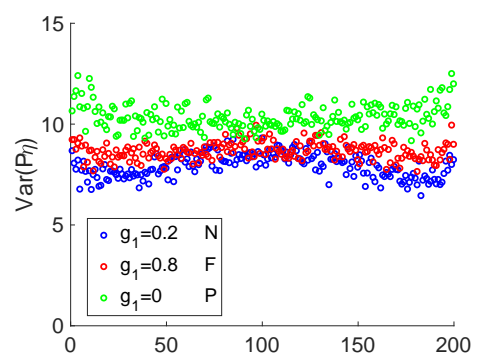

c2

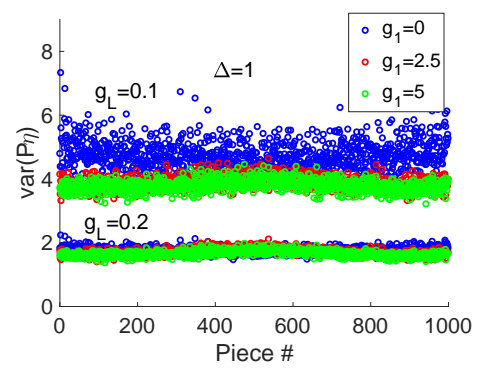

b2

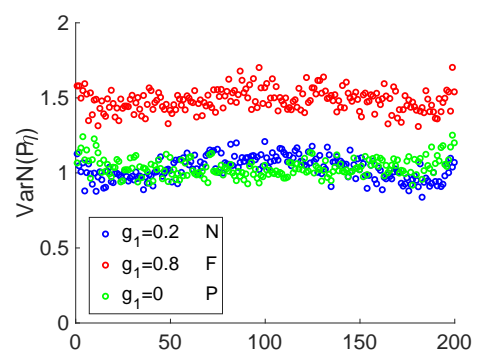

d

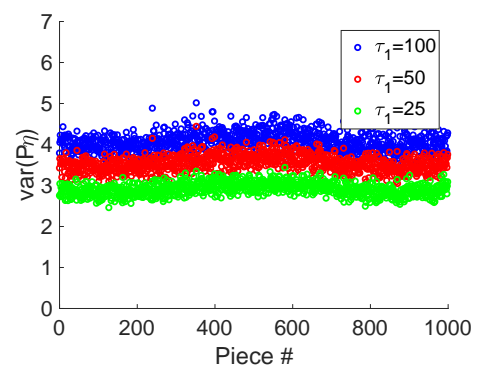

Figure 7: Variability (across trials) of the responses of linear systems to piecewise constant inputs with normally distributed amplitudes. Trials consist of different permutations of the same set of constant pieces $\left\{\eta_{k}\right\}_{k=1}^{N}$. The piecewise constant inputs $I_{\eta}$ have $\Delta=5, N=200$. a. $V$ traces for the node, focus and passive cells in response to a constant input of amplitude 1. b. Effects of changes of the linearized resonant conductance $g_{1}$ on the variances $\operatorname{Var}\left(P_{\eta}\right)$ and normalized variances $\operatorname{VarN}\left(P_{\eta}\right)$ of the peaks-and-troughs patterns. Normalized variance computed as the variance (b1) divided by the peak of the unforced cells' response to a step-constant input of amplitude 1 (a). b1. $\operatorname{Var}\left(P_{\eta}\right)$. b2. $\operatorname{VarN}\left(P_{\eta}\right)$. We used the following parameter values: $C=1, g_{L}=0.1, \tau_{1}=100, g_{1}=0.2$ (node), $g_{1}=0.8$ (focus) and $g_{1}=0$ (passive). c. Effects of changes of the linearized conductances $g_{L}$ and $g_{1}$ on the variances $\operatorname{Var}\left(P_{\eta}\right)$. c1. $\Delta=5$. c2. $\Delta=1$. We used the following additional parameter values: $C=1$ and $\tau_{1}=100$. d. Effects of changes of $\tau_{1}$ on the variances $\operatorname{Var}\left(P_{\eta}\right)$. We used $\Delta=1$. We used the following additional parameter values: $C=1, g_{L}=0.1$ and $g_{1}=2$. 
a1

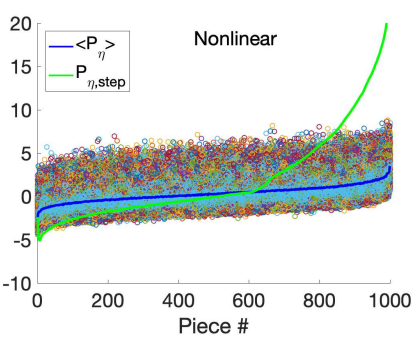

b1

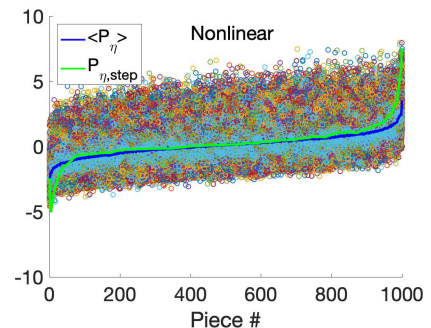

c1

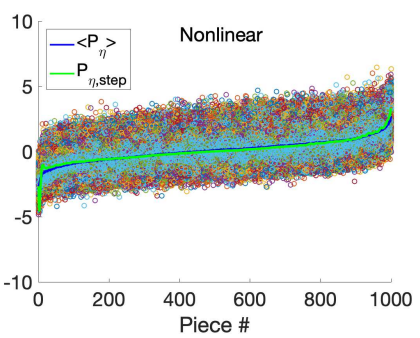

a2

\section{Passive}

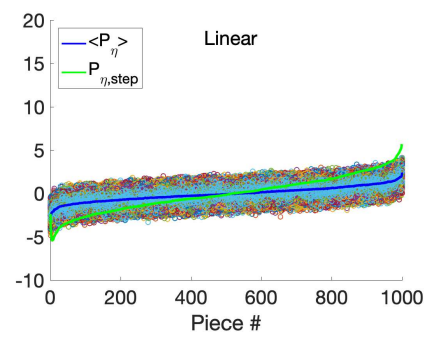

b2

\section{Node}

$\tau_{1}=100$

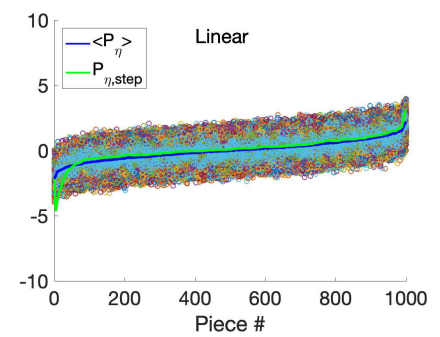

c2

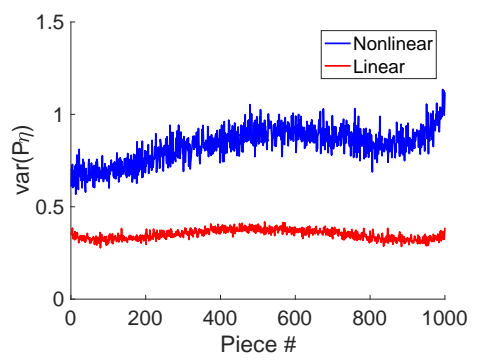

c3

\section{Node}

$\tau_{1}=10$

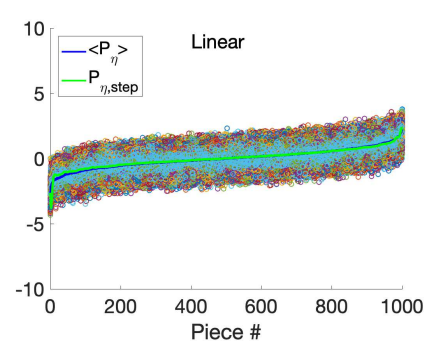

a3

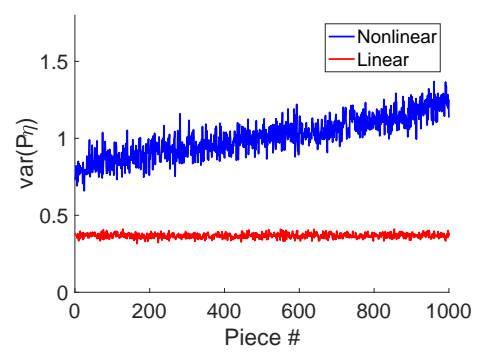

b3

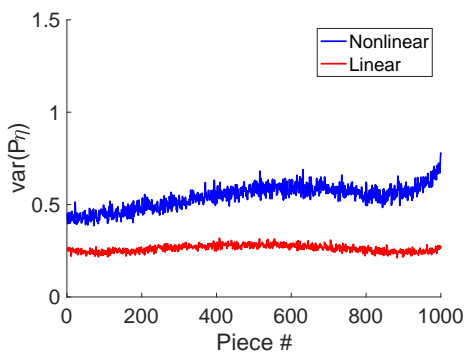

Figure 8: Effects of the model nonlinearities on the variability (across trials) in response to linear systems to piecewise constant inputs with normally distributed amplitudes. Trials consist of different permutations (1000) of the same set of constant pieces $\left\{\eta_{k}\right\}_{k=1}^{N}$. The piecewise constant inputs $I_{\eta}$ have $\Delta=1, N=1000$. The piecewise linear model is given by (3)-(4). Left and middle. The color dots indicate the peaks-and-troughs patterns for all trials reorganized so that the corresponding values of $\eta_{k}$ from which they originate are ordered in a monotonically increasing manner. All dots for a given piece correspond to the same value of $\eta_{k} .\left\langle P_{\eta}\right\rangle$ (blue) is the mean value of the reordered peaks-and-troughs patterns for each linear piece. $\left\langle P_{\eta, \text { step }}\right\rangle$ (green) is the peaks-and-troughs pattern corresponding to the (ordered) input function $I_{\eta, \text { step }}$. Right. Comparison of $\operatorname{Var}\left(P_{\eta}\right)$ between the linear (red) and piecewise linear model (blue). a. We used the following parameter values: $C=1, g_{L}=0.5, g_{1}=0, g_{c}=0.1$ and $v_{c}=0.5$. b. We used the following parameter values: $C=1, g_{L}=0.5, \tau_{1}=100, g_{1}=1, g_{c}=0.1$ and $v_{c}=0.5$. c. We used the following parameter values: $C=1, g_{L}=0.5$, $\tau_{1}=10, g_{1}=1, g_{c}=0.1$ and $v_{c}=0.5$. 
a1

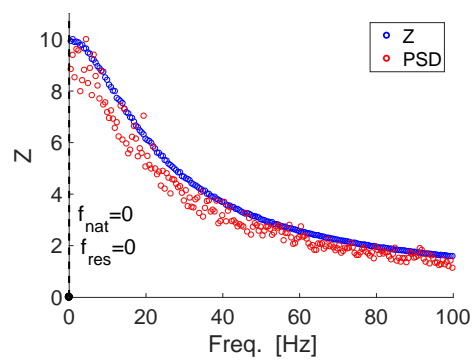

a2

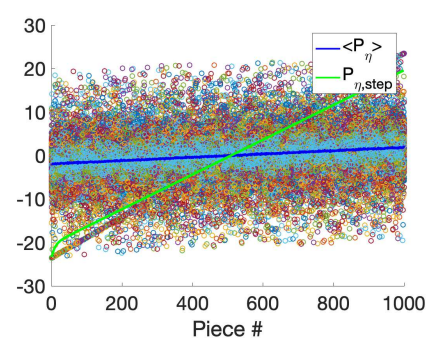

b1

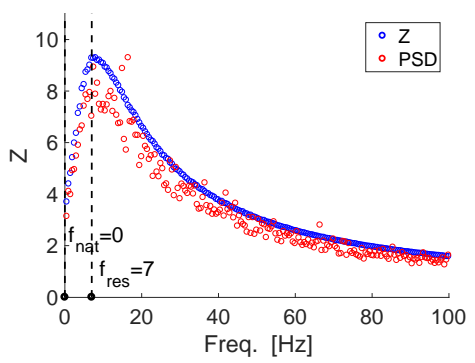

b2

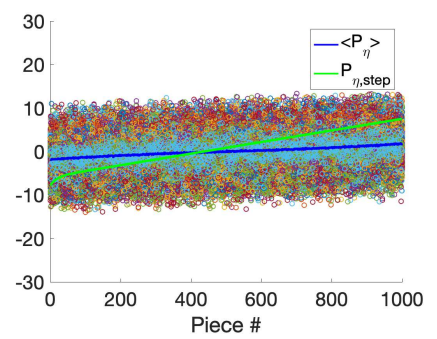

c1

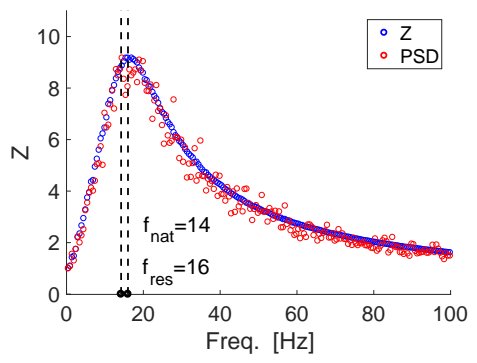

c2

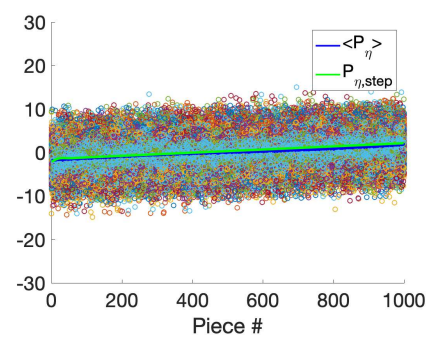

Figure 9: Piecewise constant inputs with arbitrarily ordered, but uniformly (non-randomly) distributed amplitudes capture the oscillatory properties of the target cells and the variability resulting from the autonomous transient dynamics. The piecewise constant inputs $I_{\eta}$ and $I_{\eta, s t e p}$ have constant pieces with uniformly distributed amplitudes (equal amplitude differences) in the interval $[-2,2]$ with $\Delta=1$ (total time $=1000000 \mathrm{~ms}$ for row 1 and total time $=1000 \mathrm{~ms}$ for each trial for row 2. Similar results (with less resolution) are obtained for a smaller number of pieces for row 1 (total time $=10000 \mathrm{~ms}$ ). Trials consist of different permutations (1000) of the same set of constant pieces $\left\{\eta_{k}\right\}_{k=1}^{N}$. a. $g_{1}=0$. b. $g_{1}=0.2$. c. $g_{1}=1$. We used the additional parameter values: $C=1, g_{L}=0.1$ and $\tau_{1}=100$. Top. Impedance amplitude $(Z)$ and (rescaled) power spectra density (PSD) profiles for the sample $V$ trace for the responses to $I_{\eta}$ (random) and $I_{\eta, \text { step }}$ (ordered). The PSD profiles were rescaled so that the maxima of the PSD and $Z$ profiles coincide. Bottom. The color dots indicate the peaks-and-troughs patterns for all trials reorganized so that the corresponding values of $\eta_{k}$ from which they originate are ordered in a monotonically increasing manner. All dots for a given piece correspond to the same value of $\eta_{k}$. $\left\langle P_{\eta}\right\rangle$ (blue) is the mean value of the reordered peaks-and-troughs patterns for each linear piece. $\left\langle P_{\eta, s t e p}\right\rangle$ (green) is the peaks-and-troughs pattern corresponding to the (ordered) input function $I_{\eta, \text { step }}$. 
$\Delta=1$

a1

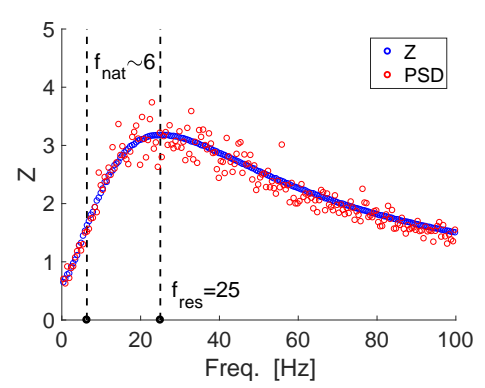

a2

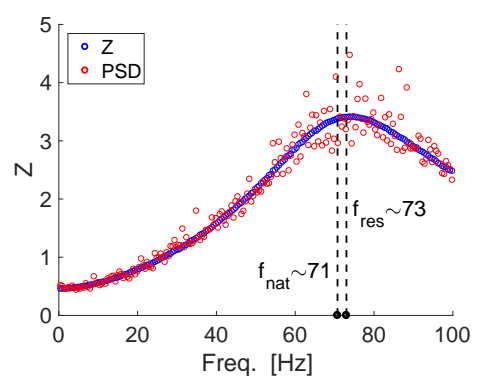

$\Delta=50$

b1i

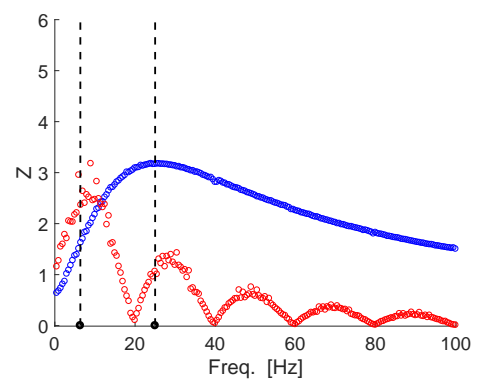

b2i

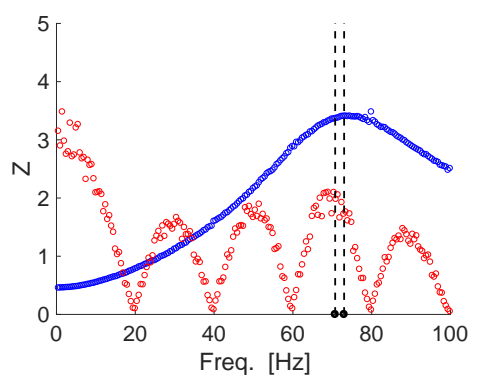

b1ii

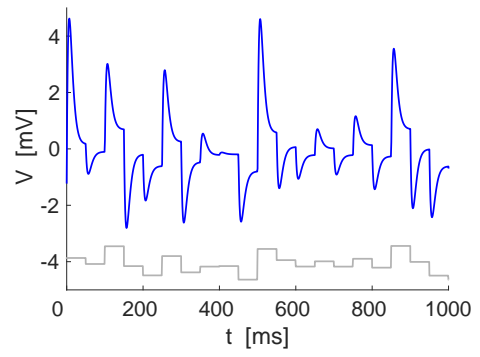

b2ii

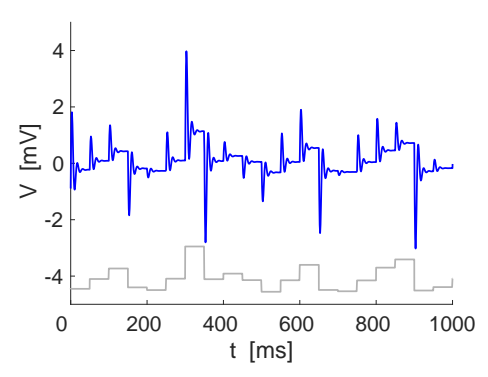

Figure 10: Cell's responses to piecewise constant inputs with normally distributed amplitudes and different constant piece durations. The piecewise constant inputs $I_{\eta}$ have a total time equal to $1,000,000$. a. $\Delta=1$. b $\Delta=50$. Top. 2D linear cell having a focus $(\mathrm{F})$ and resonance with well separated values of $f_{\text {nat }}$ and $f_{\text {res }}$. We used the following parameter values: $C=1, g_{L}=0.3, g_{1}=1.3$ and $\tau_{1}=60$. Bottom. $2 \mathrm{D}$ linear cells having a focus $(\mathrm{F})$ and resonance with similar values of $f_{\text {nat }}$ and $f_{\text {res }}$ We used the following parameter values: $C=1, g_{L}=0.2, g_{1}=2$ and $\tau_{1}=10$. Left and Middle. Superimposed $Z$ and voltage PSD profiles. The dashed vertical lines indicate $f_{\text {res }}$ (right) and $f_{\text {nat }}$ (left). Right. Sample voltage trace (blue). The gray curve is a caricature of $I_{\eta}$. 


\section{References}

[1] S. H. Strogatz. Nonlinear Dynamics and Chaos. Addison Wesley, Reading MA, 1994.

[2] E. Izhikevich. Dynamical Systems in Neuroscience: The geometry of excitability and bursting. MIT Press (Cambridge, Massachusetts), 2006.

[3] J. Guckenheimer and P. Holmes. Nonlinear oscillations, dynamical systems, and bifurcations of vector fields. Springer-Verlag, New York, 1983.

[4] J. J. Hopfield. Neural networks and physical systems with emergent collective computational abilities. Proc. Natl. Acad. Sci. USA, 79:2554-2558, 1982.

[5] D. J. Amit. Modeling brain function: The world of attractor neural networks. Cambridge University Press, New York, NY, 1989.

[6] J. J. Knierim and K. Zhang. Attractor dynamics of spatially correlated neural activity in the limbic system. Annu. Rev. Neurosci., 32:267-285, 2012.

[7] A. Samsonovich and B. L. McNaughton. Path integration and cognitive mapping in a continuous attractor neural network model. J. Neurosci., 17:5900-5920, 1997.

[8] A. D. Redish, A. N. Elga, and D. S. Touretzky. A coupled attractor model of the rodent head direction system. Network: Computation in Neural Systems, 7:671-685, 1996.

[9] C. Boucheny, N. Brunel, and A. Arleo. A continuous attractor network model without recurrent excitation: maintenance and integration in the head direction cell system. J. Comp. Neurosci., 18:205-227, 2005.

[10] K. Zhang. Representation of spatial orientation by the intrinsic dynamics of the head-direction cell ensemble: a theory. J. Neurosci., 16:2112-2126, 1996.

[11] M. Rabinovich, R. Huerta, and G. Laurent. Transient dynamics for neural processing. Science, 321:48-50, 2008.

[12] T. Nachstedt and C. Tetzlaff. Working memory requires a combination of transient and attractordominated dynamics to process unreliably timed inputs. Sci. Rep., 7:2473, 2016.

[13] G. Bondanelli and S. Ostojic. Coding with transient trajectories in recurrent neural networks. PLoS Comp. Biol., 16:e1007655, 2020.

[14] A. Robinson, H. P. C. nd Harsch. Stages of spike time variability during neuronal responses to transient inputs. Phys. Rev. E, 66:061902, 2002.

[15] M. I. Rabinovich and P. Varona. Robust transient dynamics and brain functions. Front. Comp. Neurosci., 5:24, 2011.

[16] O. Mazor and G. Laurent. Transient dynamics versus fixed points in odor representations by locust antennal lobe projection neurons. Neuron, 48:661-673, 2005.

[17] M. Stopfer, S. Bhagavan, B. H. Smith, and G. Laurent. Impared odor discrimination on desynchronization of odor-encoding neural assemblies. Nature, 390:70-74, 1997.

[18] M. J. E. Richardson, N. Brunel, and V. Hakim. From subthreshold to firing-rate resonance. J. Neurophysiol., 89:2538-2554, 2003.

[19] H. G. Rotstein. Frequency preference response to oscillatory inputs in two-dimensional neural models: a geometric approach to subthreshold amplitude and phase resonance. J. Math. Neurosci., 4:11, 2014.

[20] H. G. Rotstein. Subthreshold amplitude and phase resonance in models of quadratic type: nonlinear effects generated by the interplay of resonant and amplifying currents. J. Comp. Neurosci., 38:325-354, 2015. 
[21] B. Hutcheon, R. M. Miura, and E. Puil. Subthreshold membrane resonance in neocortical neurons. J. Neurophysiol., 76:683-697, 1996.

[22] B. Hutcheon and Y. Yarom. Resonance, oscillations and the intrinsic frequency preferences in neurons. Trends Neurosci., 23:216-222, 2000.

[23] H. G. Rotstein and F. Nadim. Frequency preference in two-dimensional neural models: a linear analysis of the interaction between resonant and amplifying currents. J. Comp. Neurosci., 37:9-28, 2014.

[24] N. G. Van Kampen. Stochastic Processes in Physics and Chemistry. North-Holland Personal Library, 2011.

[25] G. Deco, E. Rolls, and R. Romo. Stochastic dynamics as a principle of brain function. Prog. Neurobiol., 88:1-16, 2009.

[26] A. A. Faisal, L. P. J. Selen, and D. M. Wolpert. Noise in the nervous system. Nat. Rev. Neurosci., 9:292-303, 2008.

[27] J. H. Shalinsky, J. Magistretti, L. Ma, and A. A. Alonso. Muscarinic activation of a cation current and associated current noise in entorhinal-cortex layer-II neurons. J. Neurophysiol., 88:1197$1211,2002$.

[28] J. A. White and J. S. Haas. Intrinsic noise from voltage-gated ion channels: Effects on dynamics and reliability in intrinsically oscillatory neurons. In Handbook of Biological Physics, 4:257-278, 2001.

[29] A. D. Jr. Dorval and J. A. White. Channel noise is essential for perithreshold oscillations in entorhinal stellate neurons. J. Neurosci., 25:10025-10028, 2005.

[30] L. J. DeFelice. Introduction to channel noise. Plenum Press, 1981.

[31] C. C. Chow and J. A. White. Spontaneous action potentials due to channel fluctuation. Biophysical J., 71:3013-3021, 1996.

[32] J. White, J. Rubinstein, and A. Kay. Channel noise in neurons. Trends Neurosci., 23:131-137, 2000.

[33] E. Schneidman, B. Freedman, and I. Segev. Ion channel stochasticity may be critical in determining the reliability and precision of spike timing. Neural Computat., 10:1679-1703, 1998.

[34] F. J. Sigworth. The variance of sodium current fluctuations at the node of ranvier. J. Physiol (Lond.), 307:97-129, 1980.

[35] W. H. Calvin and C. F. Stevens. Synaptic noise as a source of variability in the interval between action potentials. Science, 155:842-844, 1967.

[36] N. Brunel, F. S. Chance, N. Fourcaud, and L. F. Abbott. Effects of synaptic noise and filtering on the frequency response of spiking neurons. Phys. Rev. Lett., 86:2186-2189, 2001.

[37] A. Destexhe, M. Badoual, Z. Piwkowska, T Bal, and M. Rudolph. A novel method for characterizing synaptic noise in cortical neurons. Neurocomputing, 58:191-196, 2004.

[38] J. M. Fellous, M. Rudolph, A. Destexhe, and T. J. Sejnowski. Synaptic background noise controls the input/output characteristics of single cells in an in vitro model of in vivo activity. Neurosci., 122:811-829, 2003.

[39] Y. Yarom and J. Hounsgaard. Voltage fluctuations in neurons: Signal or noise? Physiological Reviews, 91:917-929, 2011.

[40] S. Hong, S. Ratté, S. A. Prescott, and E. De Schutter. Single neuron firing properties impact correlation-based population coding. J. Neurosci., 32:1413-1428, 2012.

[41] R. F. O. Pena, M. A. Zaks, and A. C. Roque. Dynamics of spontaneous activity in random networks with multiple neuron subtypes and synaptic noise. J. Comp. Neurosci., 45:1-28, 2018. 
[42] A. Destexhe and M. Rudolph-Lilith. Neuronal Noise. Springer, 2012.

[43] B. Marin, R. D. Pinto, R. C. Elson, and E. Colli. Noise, transient dynamics, and the generation of realistic interspike interval variation in square-wave burster neurons. Phys. Rev. E, 90:042718, 2014.

[44] J. Lee and J. Lee. Quantitative analysis of a transient dynamics of a gene regulatory network. Phys. Rev. E, 98:062404, 2018.

[45] H. G. Rotstein, T. Oppermann, J. A. White, and N. Kopell. The dynamic structure underlying subthreshold oscillatory activity and the onset of spikes in a model of medial entorhinal cortex stellate cells. J. Comp. Neurosci., 21:271-292, 2006.

[46] Stochastic resonance in climatic change. Benzi, r. and parisi, g. and sutera, a. and vulpiani, a. Tellus, 34:10-16, 1982.

[47] L. Gammaitoni, P. Hanggi, P. Jung, and Marchesoni. F. Stochastic resonance. Rev. Mod. Phys., 70:223-287, 1998.

[48] B. McNamara and K. Wiesenfeld. Theory of stochastic resonance. Phys. Rev. A, 39:48544869, 1989.

[49] G. Mato. Stochastic resonance using noise generated by a neural network. Phys. Rev. E, 59:3339-3343, 1989.

[50] M. D. McDonnell and D. Abboott. What is stochastic resonance? definitions, misconceptions, debates, and its relevance to biology. PLoS Comp. Biol., 5:e1000348, 2009.

[51] J. K. Douglass, L. Wilkens, E. Pantazelou, and F. Moss. Noise enhancement of information transfer in crayfish mechanoreceptors by stochastic resonance. Nature, 365:337-340, 1993.

[52] K. Wiesenfeld and F Moss. Stochastic resonance and the benefits of noise: from ice ages to crayfish and SQUIDs. Nature, 373:33-36, 1995.

[53] J. J. Collins, T. T. Imhoff, and P. Grigg. Noise-enhanced information transmission in rat SA1 cutaneous mechanoreceptors via aperiodic stochastic resonance. J. Neurophysiol., 76:642645, 1996.

[54] C. B. Muratov, E. Vanden-Eijnden, and W. E. Self-induced stochastic resonance in excitable systems. Physica D, 210:227-240, 2005.

[55] A. S. Pikovsky and J. Kurths. Coherence resonance in a noise-driven excitable system. Phys. Rev. Lett., 78:775-778, 1997.

[56] B. Lindner, J. García-Ojalvo, A. Neiman, and L. Schimansky-Geier. Effects of noise in excitable systems. Physics Reports, 392:321-424, 2004.

[57] A. Neiman, P. I. Saparin, and L. Stone. Coherence resonance at noisy precursors of bifurcations in nonlinear dynamical systems. Phys. Rev. E, 56:270-273, 1997.

[58] S.-G. Lee, A. Neiman, and S. Kim. Coherence resonance in a hodgkin-huxley neuron. Phys. Rev. E, 57:3292-3297, 1998.

[59] J. R. Pradines, G. V. Osipov, and J. J. Collins. Coherence resonance in excitable and oscillatory systems: The essential role of slow and fast dynamics. Phys. Rev. E, 60:6407-6410, 1999.

[60] T. Tateno and K. Pakdaman. Random dynamics of the morris-lecar neural model. Chaos, 14:511-530, 2004.

[61] E. DeVille, R. E. L. andVanden-Eijnden and C. B. Muratov. Two distinct mechanisms of coherence in randomly perturbed dynamical systems. Phys. Rev. E, 72:031105, 2005.

[62] E. Baspinar, L. Schulen, S. Olmi, and A. Zakharova. Coherence resonance in neuronal populations: Mean-field versus network model. Phys. Rev. E, 103:032308, 2021. 
[63] H. Gang, T. Ditzinger, C. Z. Ning, and H. Haken. Stochastic resonance without periodic forcing. Phys. Rev. Lett., 71:807-810, 1993.

[64] K. Wiesenfeld. Noisy precursors of nonlinear instabilities. J. Stat. Phys., 38:1071-1097, 1985.

[65] D. Sigeti and Horsthemke. Pseudo-regular oscillations induced by external noise. J. Stat. Phys., 54:1217-1222, 1989.

[66] K. Matsumoto and I. Tsuda. Noise-induced order. J. Stat. Phys., 31:87-106, 1983.

[67] V. S. Anishchenko and B. Neiman. Stochastic synchronization. Stochastic Dynamics (in Lecture Notes Physics), 484:154-166, 1997.

[68] A. S. Pikovsky. Synchronization and stochastization of nonlinear oscillations by external noise. In:Nonlinear and Turbulent Processes in Physics, ed. R.Z.Sagdeev, Harwood Acad. Publ., 3:1601-1604, 1984.

[69] V. Hakim and W.-J. Rappel. Noise-induced periodic behaviour in the globally coupled complex Ginzburg-Landau equation. Europhys. Lett., 27:637-642, 1994.

[70] C. Kurrer and K. Schulten. Noise-induced synchronous neuronal oscillations. Phys. Rev. E, 51:6213-6218, 1995.

[71] W. J. Rappel and A. Karma. Noise-induced coherence in neural networks. Phys. Rev. Lett., 77:3251-3259, 1996.

[72] J. Pham, K. Pakdaman, and J.-F. Vibert. Noise-induced coherent oscillations in randomly connected neural networks. Phys. Rev. E, 58:3610-3622, 1998.

[73] R. V. Jensen. Synchronization of randomly driven nonlinear oscillators. Phys. Rev. E, 58:R6907-R6910, 1998.

[74] S. Lim and J. Rinzel. Noise-induced transitions in slow wave neuronal dynamics. J. Comp. Neurosci., 28:1-17, 2010.

[75] D. Levenstein, G. Buzsaki, and J. Rinzel. Nrem sleep in the rodent neocortex and hippocampus reflects excitable dynamics. Nature Comm., 10:2478, 2019.

[76] A. Longtin, A. Bulsara, and F. Moss. Time-interval sequences in bistable systems and the noise-induced transmission of information by sensory neurons. Phys. Rev. Lett., 67:656, 1991.

[77] J. A. White, R. Klink, A. Alonso, and A. R. Kay. Noise from voltage-gated ion channels may influence neuronal dynamics in the entorhinal cortex. J. Neurophysiol., 80:262-269, 1998.

[78] P. F. Rowat and R. C. Elson. State-dependent effects of Na channel noise on neuronal burst generation. J. Comp. Neurosci., 16:87-112, 2004.

[79] J. P. Baltanas and J. M. Casado. Bursting behavior of the Fitzhugh-Nagumo neuron model subject to quasi-monochromatic noise. Physica D, 122:231-240, 1998.

[80] J. J. Collins, C. C. Chow, and T. T. Imhoff. Aperiodic stochastic resonance in excitable systems. Phys. Rev. E, 76:642-645, 1995.

[81] G. E. Uhlenbeck and L. S. Ornstein. On the theory of brownian motion. Phys. Rev., 36:823841, 1930.

[82] C. W. Gardiner. Handbook of Stochastic Methods. Springer-Verlag, Berlin, 1985.

[83] H. Risken. The Fokker-Planck equation, 2nd ed. Springer-Verlag, Berlin, 1989.

[84] E. J. Allen, S. J. Novosel, and Z. Zhang. Finite element and difference approximation of some linear stochastic partial differential equations. Stochastics and Stochastic Reports, 64:117$142,1998$.

[85] P. J. Thomas and B. Lindner. Phase descriptions of a multidimensional Ornstein-Uhlenbeck process. Phys. Rev. E, 99:062221, 2019. 
[86] Q. Du and T. Zhang. Numerical approximation of some linear stochastic partial differential equations driven by special additive noise. SIAM J. Numer. Anal., 400:1421-1445, 2002.

[87] R. L. Burden and J. D. Faires. Numerical analysis. PWS Publishing Company - Boston, 1980.

[88] J. Middleton, M. Chacron, B. Lindner, and A. Longtin. Firing statistics of a neuron model driven by long-range correlated noise. Phys. Rev. E, 68:021920, 2003.

[89] M. J. E. Richardson. Spike-train spectra and network response functions for non-linear integrate-and-fire neurons. Biol. Cybern., 99:381-392, 2008.

[90] C. Laing and G. J. Lord. Stochastic methods in neuroscience. Oxford University Press, 2010.

[91] R. Fernandez and J. A. White. Artificial synaptic conductances reduce subthreshold oscillations and periodic firing in stellate cells of the entorhinal cortex. J. Neurosci., 28:3790-3803, 2008.

[92] A. A. Sharp, M. B. O'Neil, L. F. Abbott, and E. Marder. The dynamic clamp: artificial conductances in biological neurons. Trends Neurosci., 16:389-394, 1993.

[93] A. A. Prinz, V. Thirumalai, and E. Marder. The functional consequences of changes in the strength and duration of synaptic inputs to oscillatory neurons. J. Neurosci., 23:943-954, 2003.

[94] F. Zhang, L.-P. Wang, M. Brauner, J. F. Lewwald, K. Kay, N. Watzke, P. G. Wookd, E. Bamberg, G. Nagel, A. Gottschalk, and K. Deisseroth. Multimodal fast optical interrogation of neural circuitry. Nature, 446:633-641, 2007.

[95] K. Deisseroth. Optogenetics. Nat. Methods, 8:26-29, 2011.

[96] J. G. Bernstein and E. S. Boyden. Optogenetic tools for analyzing the neural circuits of behavior. Curr. Opin. Neurobiol., 22:61-71, 2012.

[97] A. Renart and C. K. Machens. Variability in neural activity and behavior. Curr. Opin. Neurobiol., 25:211-220, 2014.

[98] M. M. Churchland, B. M. Yu, J. P. Cunningham, L. P. Sugrue, M. R. Cohen, G. S. Corrado, W. T. Newsome, A. M. Clark, P. Hosseini, B. B. Scott, D. C. Bradley, M. A. Smith, A. Kohn, J. A. Movshon, K. M. Armstrong, T. Moore, S. W. Chang, L. H. Snyder, S. G. Lisberger, N. J. Priebe, I. M. Finn, D. Ferster, S. I. Ryu, G. Santhanam, M. Sahani, and K. V. Shenoy. Stimulus onset quenches neural variability: a widespread cortical phenomenon. Nature Neurosci., 13:369$378,2010$.

[99] T. Ito, S. L. Brincat, R. D. Mil, M. Siegel, B. J. He, E. K. Miller, H. G. Rotstein, and M. W. Cole. Task-evoked activity quenches neural correlations and variability in large-scale brain systems. PLoS Comp. Biol., 16:e1007983, 2020.

\section{A Intrinsic and resonant oscillatory properties of 2D linear sys- tems}

Consider

$$
\left\{\begin{array}{l}
x^{\prime}=a x+b y+A_{i n} e^{i \omega t} \\
y^{\prime}=c x+d y
\end{array}\right.
$$

where $a, b, c$ and $d$ are constants, $\omega=2 \pi f / 1000>0$ is the input frequency and $A_{\text {in }} \geq 0$ is the input amplitude. The prime sign represents the derivative with respect to $t$. The units of $t$ are $\mathrm{ms}$ and the units of $f$ are $\mathrm{Hz}$. 


\section{A.1 Intrinsic oscillations}

The characteristic polynomial for the corresponding homogeneous system $\left(A_{i n}=0\right)$ is given by

$$
r^{2}-(a+d) r+(a d-b c)=0 .
$$

The eigenvalues are given by

$$
r_{1,2}=\frac{a+d \pm \sqrt{(a-d)^{2}+4 b c}}{2},
$$

and the natural (intrinsic) frequency of the (damped) oscillations (in $\mathrm{Hz}$ if $t$ has units of $\mathrm{ms}$ ) is given by

$$
f_{\text {nat }}=\frac{\sqrt{-(a-d)^{2}-4 b c}}{4 \pi} 1000
$$

assuming $(a-d)^{2}+4 b c<0$.

\section{A.2 Resonance and the impedance amplitude profile}

The impedance amplitude profile $Z(\omega)$ for system (10)-(11) is the magnitude

$$
Z(\omega)=\sqrt{\frac{d^{2}+\omega^{2}}{\left(a d-b c-\omega^{2}\right)^{2}+(a+d)^{2} \omega^{2}}}
$$

of the complex valued coefficient of the particular solution to the system

$$
\mathbf{Z}(\omega)=\frac{(-d+i \omega)}{(-a+i \omega)(-d+i \omega)-b c} .
$$

For 1D system, these quantities are given, respectively, by

$$
Z(\omega)=\frac{1}{\sqrt{a^{2}+\omega^{2}}}
$$

and

$$
\mathbf{Z}(\omega)=\frac{1}{(-a+i \omega)} .
$$

The resonance frequency $f_{r e s}$ (in $\mathrm{Hz}$ if $t$ has units of $\mathrm{ms}$ ) is the frequency at which $Z$ reaches its maximum

$$
f_{\text {res }}=\frac{\sqrt{-d^{2}+\sqrt{b^{2} c^{2}-2 a b c d-2 d^{2} b c}}}{2 \pi} 1000
$$

\section{A.3 Response to constant inputs}

The equilibrium solution to system (10) for a constant input $A_{\text {in }}$ (i.e., $\omega=0$ ) is given by

$$
\bar{x}=-\frac{A_{\text {in }} d}{a d-b c} \quad \text { and } \quad \bar{y}=\frac{A_{\text {in }} c}{a d-b c} .
$$

The eigenvectors are given by

$$
z_{1,2}=\left[b\left(r_{1,2}-a\right)\right]^{T}
$$


The solution satisfying the initial conditions $[x(0) \quad y(0)]^{T}=\left[\begin{array}{ll}x_{0} & y_{0}\end{array}\right]^{T}$ is given by

$$
\left[\begin{array}{l}
x \\
y
\end{array}\right]=c_{1}\left[\begin{array}{c}
b \\
r_{1}-a
\end{array}\right] e^{r_{1} t}+c_{2}\left[\begin{array}{c}
b \\
r_{2}-a
\end{array}\right] e^{r_{2} t}+\left[\begin{array}{l}
\bar{x} \\
\bar{y}
\end{array}\right]
$$

where

$$
c_{1}=\frac{b\left(y_{0}-\bar{y}\right)-\left(x_{0}-\bar{x}\right)\left(r_{2}-a\right)}{b\left(r_{1}-r_{2}\right)} \quad \text { and } \quad c_{2}=\frac{-b\left(y_{0}-\bar{y}\right)+\left(x_{0}-\bar{x}\right)\left(r_{1}-a\right)}{b\left(r_{1}-r_{2}\right)}
$$

For 1D systems $(b=0)$,

$$
\bar{x}=-\frac{A_{\text {in }}}{a}
$$

and

$$
x=\left(x_{0}-\frac{A_{\text {in }}}{a}\right) e^{a t}-\frac{A_{\text {in }}}{a},
$$

where $x(0)=x_{0}$

\section{B Ornstein-Uhlenbeck (OU) Process}

\section{B.1 One-dimensional OU process}

The 1D OU process [81] is described by the following linear stochastic differential equation

$$
X^{\prime}=-a X+I+\sigma \eta(t)
$$

where $a>0, I$ and $\sigma$ are constants and $\eta(t)$ is zero-mean and $\delta$-correlated Gaussian white noise. The parameter $a$ is the inverse of the time constant and measure the strength by which the system reacts to perturbations. The parameter $\sigma$ measures the intensity of the noise. The quotient $I / \alpha$ is the asymptotic mean.

Using standard methods $[82,83]$ one can compute the solution satisfying $X(0)=x_{0}$, which is the sum of a deterministic function with the form (24) and an integral of a deterministic function with respect to a Wiener process. The solution is normally distributed with mean and variance given, respectively by

$$
E[X(t)]=\left(X_{0}-\frac{I}{a}\right) e^{-a t}+\frac{I}{a}
$$

and

$$
\operatorname{Var}[X(t)]=\frac{\sigma^{2}}{2 a}\left(1-e^{-2 a t}\right)
$$

\section{B.2 Higher-dimensional OU process}

The multivariate $\mathrm{OU}$ process [81] is described by the following linear stochastic differential equation

$$
X^{\prime}=A X+B+\Sigma H(t)
$$

where $X$ is an $n$-dimensional vector, $A$ is an $n \times n$ matrix, $B$ is an $n$-dimensional vector, $\Sigma$ is an $n \times m$ matrix and $H$ is a vector of independent zero-mean and $\delta$-correlated Gaussian white noise 
components. Using standard methods $[82,83]$ one can compute the solution satisfying $X(0)=x_{0}$. The solution is normally distributed. The mean is given by

$$
E[X(t)]=\left[e^{A t}-B\right] A^{-1} B+e^{A t} X(0),
$$

and the covariance matrix is given by

$$
\operatorname{Cov}[X(t)]=\int_{0}^{t} e^{A s} \Sigma \Sigma^{T} e^{A^{T} s}
$$

Under certain conditions, the covariance matrix corresponding to the stationary solutions reads

$$
\operatorname{Cov}[X(t)]=-\frac{\Sigma \Sigma^{T}}{2 \operatorname{Tr}(A)}-\frac{[A-\operatorname{Tr}(A) I] \Sigma \Sigma^{T}[A-\operatorname{Tr}(A) I]^{T}}{2 \operatorname{Tr}(A) \operatorname{det}(A)} .
$$




\section{Supplementary Material}

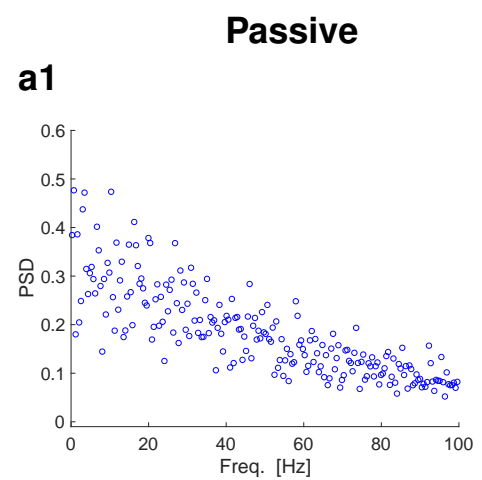

a2

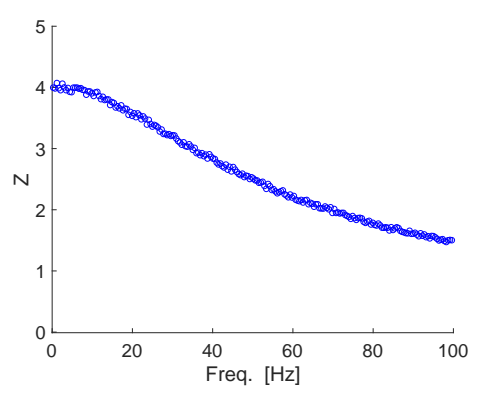

Node

\section{b1}

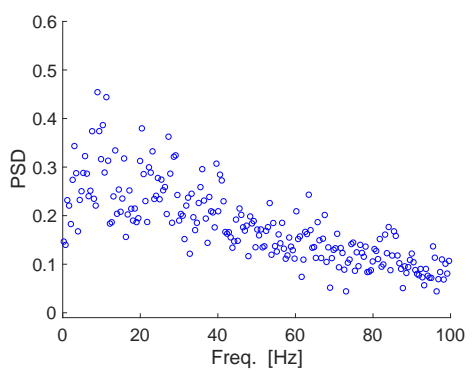

b2

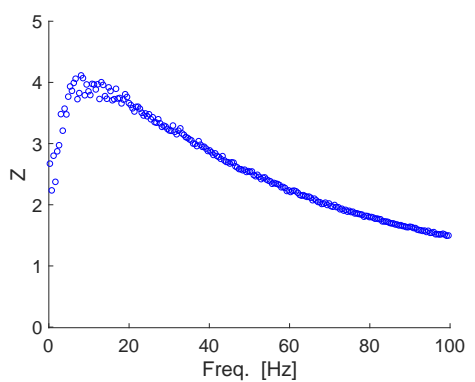

\section{Focus}

\section{c1}

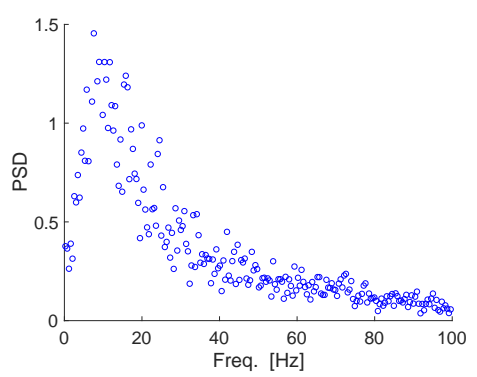

c2

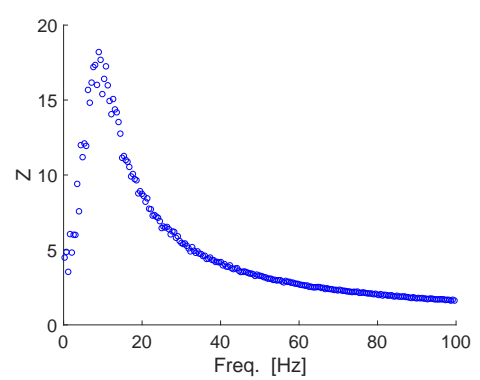

Figure S1: Piecewise constant inputs with arbitrarily ordered, but uniformly (non-randomly) distributed amplitudes capture the transient dynamics of the target cells. The piecewise constant inputs $I_{\eta}$ have $\Delta=5$ (total time $=10000 \mathrm{~ms}, \mathrm{~N}$ = 2000). The parameter values are the same as in Figs. 1 and 2. Row 1. Power spectra density (PSD) profiles for the sample $V$ trace. Row 2. Impedance amplitude $(Z)$ profiles for the sample $V$ trace. a. Passive cell $\left(f_{\text {nat }}=f_{\text {res }}=0\right)$. We used the following paramete.r values: $C=1, g_{L}=0.25$. b. $2 \mathrm{D}$ linear system exhibiting an overshoot in response to step-constant inputs $\left(\left(f_{\text {nat }}=0, f_{\text {res }} \sim 9 \mathrm{~Hz}\right)\right.$. We used the following parameter values: $C=1, g_{L}=0.25, g_{1}=0.25, \tau_{1}=100$. c. $2 \mathrm{D}$ linear system exhibiting damped oscillations in response to step-constant inputs $\left(f_{\text {nat }} \sim f_{\text {res }} \sim 8 H z\right)$. We used the following parameter values: $C=1, g_{L}=0.05, g_{1}=0.3, \tau_{1}=100$ 


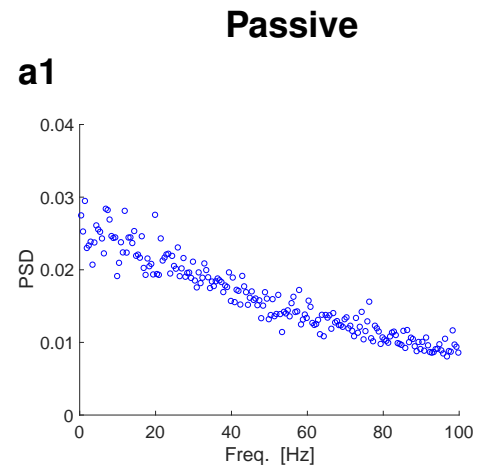

a2

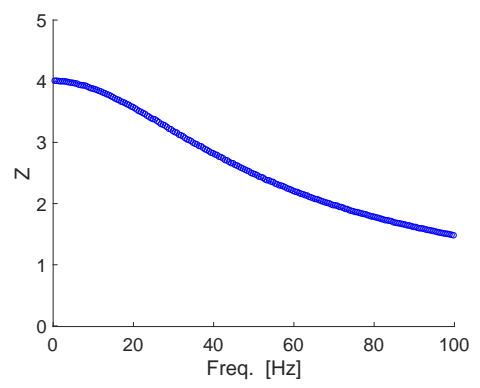

Node

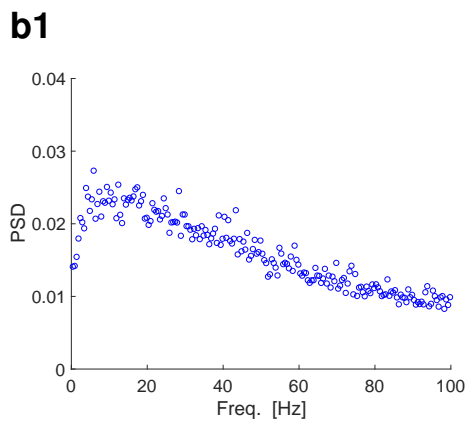

b2

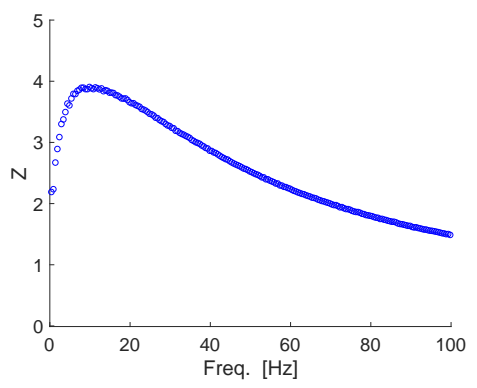

Focus

c1

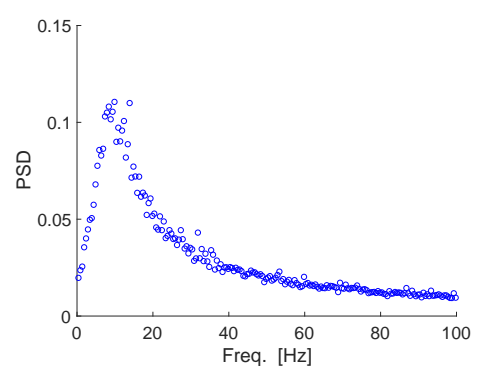

c2

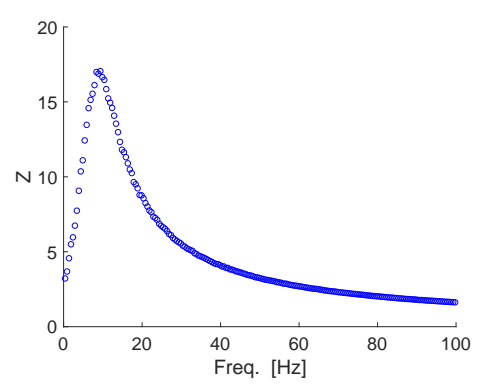

Figure S2: Piecewise constant inputs with arbitrarily ordered, but uniformly (non-randomly) distributed amplitudes capture the transient dynamics of the target cells. The piecewise constant inputs $I_{\eta}$ have $\Delta=1$ (total time $=1000000$ $\mathrm{ms}, \mathrm{N}=1000000$ ). The parameter values are the same as in Figs. 1 and 2. Row 1. Power spectra density (PSD) profiles for the sample $V$ trace. Row 2. Impedance amplitude $(Z)$ profiles for the sample $V$ trace. a. Passive cell $\left(f_{\text {nat }}=f_{\text {res }}=0\right)$. We used the following paramete.r values: $C=1, g_{L}=0.25$. b. $2 \mathrm{D}$ linear system exhibiting an overshoot in response to step-constant inputs $\left(\left(f_{\text {nat }}=0, f_{\text {res }} \sim 9 H z\right)\right.$. We used the following parameter values: $C=1, g_{L}=0.25, g_{1}=0.25$, $\tau_{1}=100$. c. $2 \mathrm{D}$ linear system exhibiting damped oscillations in response to step-constant inputs $\left(f_{\text {nat }} \sim f_{\text {res }} \sim 8 \mathrm{~Hz}\right)$. We used the following parameter values: $C=1, g_{L}=0.05, g_{1}=0.3, \tau_{1}=100$ 
a1

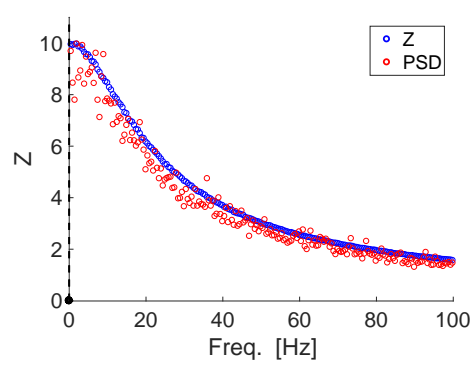

a2

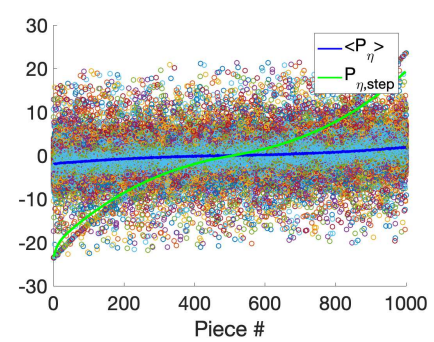

b1

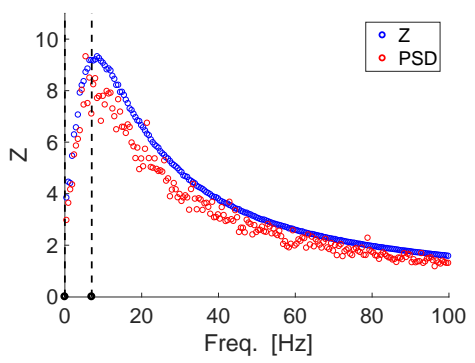

b2

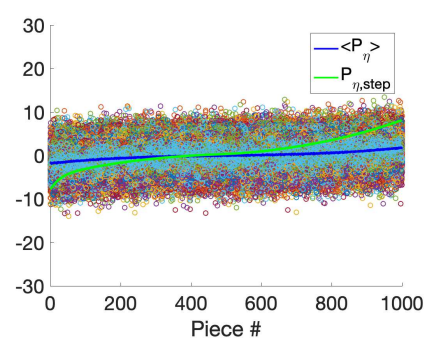

c1

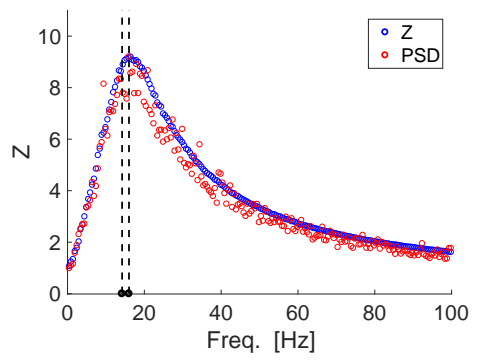

c2

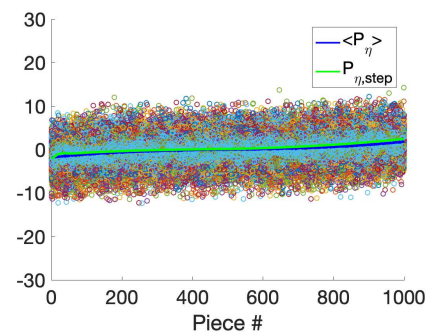

Figure S3: Piecewise constant inputs with arbitrarily ordered, but bell-shaped (non-randomly) distributed amplitudes capture the oscillatory properties of the target cells and the variability resulting from the autonomous transient dynamics. The piecewise constant inputs $I_{\eta}$ and $I_{\eta, \text { step }}$ have constant pieces with (non-random) bell shapped distributed amplitudes in the interval $[-2,2]$ with $\Delta=1$ (total time $=1000000 \mathrm{~ms}$ for row 1 and total time $=1000 \mathrm{~ms}$ for each trial for row 2. Similar results (with less resolution, variance $=1.5$ ) are obtained for a smaller number of pieces for row 1 (total time $=10000 \mathrm{~ms})$. Trials consist of different permutations (1000) of the same set of constant pieces $\left\{\eta_{k}\right\}_{k=1}^{N}$. a. $g_{1}=0$. b. $g_{1}=0.2$. c. $g_{1}=1$. We used the additional parameter values: $C=1, g_{L}=0.1$ and $\tau_{1}=100$. Top. Impedance amplitude $(Z)$ and (rescaled) power spectra density (PSD) profiles for the sample $V$ trace for the responses to $I_{\eta}$ (random) and $I_{\eta, s t e p}$ (ordered). The PSD profiles were rescaled so that the maxima of the PSD and $Z$ profiles coincide. Bottom. The color dots indicate the peaks-and-troughs patterns for all trials reorganized so that the corresponding values of $\eta_{k}$ from which they originate are ordered in a monotonically increasing manner. All dots for a given piece correspond to the same value of $\eta_{k} .\left\langle P_{\eta}\right\rangle$ (blue) is the mean value of the reordered peaks-and-troughs patterns for each linear piece. $\left\langle P_{\eta, s t e p}\right\rangle$ (green) is the peaks-and-troughs pattern corresponding to the (ordered) input function $I_{\eta, \text { step }}$. 
a1

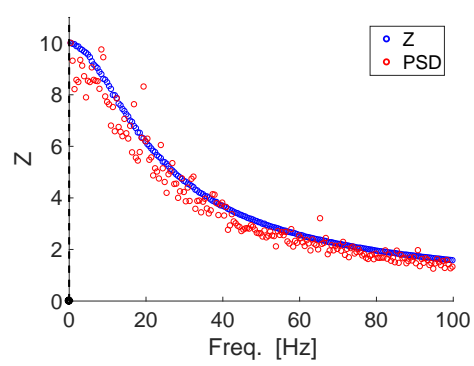

a2

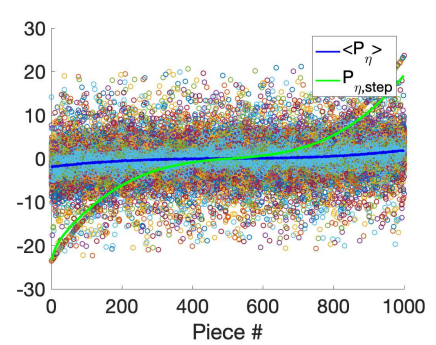

b1

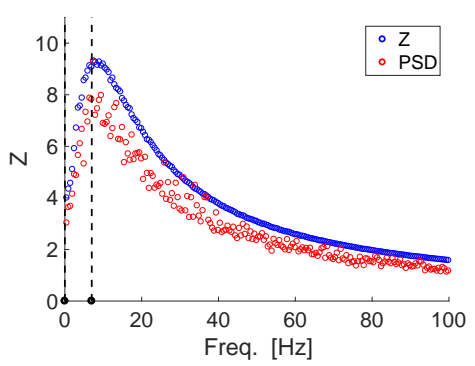

b2

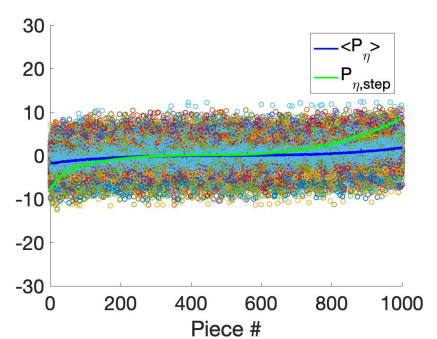

c1

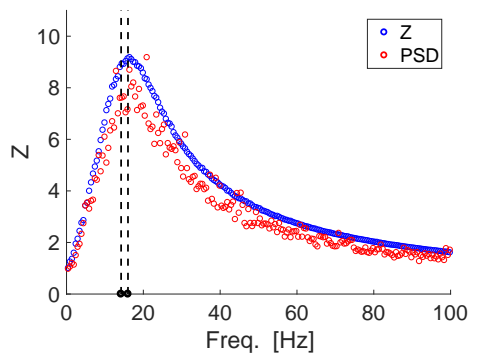

c2

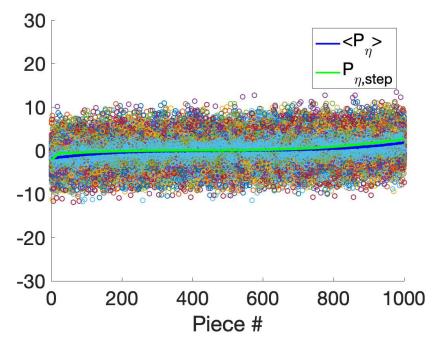

Figure S4: Piecewise constant inputs with arbitrarily ordered, but bell-shaped (non-randomly) distributed amplitudes capture the oscillatory properties of the target cells and the variability resulting from the autonomous transient dynamics. The piecewise constant inputs $I_{\eta}$ and $I_{\eta, \text { step }}$ have constant pieces with (non-random) bell shapped distributed amplitudes in the interval $[-2,2]$ with $\Delta=1$ (total time $=1000000 \mathrm{~ms}$ for row 1 and total time $=1000 \mathrm{~ms}$ for each trial for row 2. Similar results (with less resolution, variance $=1$ ) are obtained for a smaller number of pieces for row 1 (total time $=10000 \mathrm{~ms}$ ). Trials consist of different permutations (1000) of the same set of constant pieces $\left\{\eta_{k}\right\}_{k=1}^{N}$. a. $g_{1}=0$. b. $g_{1}=0.2$. c. $g_{1}=1$. We used the additional parameter values: $C=1, g_{L}=0.1$ and $\tau_{1}=100$. Top. Impedance amplitude $(Z)$ and (rescaled) power spectra density (PSD) profiles for the sample $V$ trace for the responses to $I_{\eta}$ (random) and $I_{\eta, \text { step }}$ (ordered). The PSD profiles were rescaled so that the maxima of the PSD and $Z$ profiles coincide. Bottom. The color dots indicate the peaks-and-troughs patterns for all trials reorganized so that the corresponding values of $\eta_{k}$ from which they originate are ordered in a monotonically increasing manner. All dots for a given piece correspond to the same value of $\eta_{k}$. $\left\langle P_{\eta}\right\rangle$ (blue) is the mean value of the reordered peaks-and-troughs patterns for each linear piece. $\left\langle P_{\eta, \text { step }}\right\rangle$ (green) is the peaks-and-troughs pattern corresponding to the (ordered) input function $I_{\eta, \text { step }}$. 